Sportwiss $2011 \cdot 41: 216-232$

DOI 10.1007/s12662-011-0198-4

(c) Springer-Verlag 2011
Claudia Klostermann - Siegfried Nagel

Institut für Sportwissenschaft, Universität Bern

\title{
Sport treiben ein Leben lang?
}

\section{Einfluss der Sportkarriere der 1. Lebenshälfte auf das Sportengagement im mittleren und späten Erwachsenenleben}

\section{Hintergrund}

Angesichts der steigenden Lebenserwartung und der Befürchtung, dass die gewonnenen Lebensjahre mit Krankheit und Pflegebedürftigkeit verbunden sind, rückt die Frage nach den Bedingungen für ein gesundes und erfolgreiches Altern in den Mittelpunkt wissenschaftlichen Interesses. Dazu kann der Sport einen wichtigen Beitrag leisten (u. a. Lippke, Schwarzer \& Fuchs, 2001; Möller, 1999). Trotz dieser Erkenntnis über die positiven Wirkungen einer körperlich-sportlichen Aktivität im Alter ist bei älteren Menschen im Vergleich zu jüngeren Altersgruppen eine geringere Sportbeteiligung zu konstatieren (u. a. Breuer, 2003, S. 267; Breuer, 2004, S. 53; Becker \& Klein, 2007, S. 297), wenngleich die Mitgliederzahlen der über 6o-Jährigen im Deutschen Olympischen Sportbund (DOSB) in den vergangenen Jahren stetig angestiegen sind. Vor dem Hintergrund dieser Beobachtungen stellt sich die Frage, welche Faktoren ein (mögliches) Sportengagement in der 2. Lebenshälfte beeinflussen.

Hierbei ist eine Vielzahl psychologischer, sozialer und physischer Einflussfaktoren in Betracht zu ziehen. Die Zielgruppe der Menschen in der 2. Lebenshälfte zeichnet sich durch vielfältige Lebenserfahrungen aus. Nach Allmer (1992, S. 38) lässt sich „die Zuwendung zur sportlichen Aktivität im Alter (...) überhaupt erst verständlich machen, wenn sie unter Berücksichtigung der bisheri- gen sportbezogenen Entwicklung interpretiert wird“. Hierbei ist davon auszugehen, dass Personen im mittleren und späteren Erwachsenenalter im Verlauf ihres Lebens in irgendeiner Form mit Sport bereits in Kontakt gekommen sind, wenngleich diese sportbezogenen Erfahrungen individuell sehr unterschiedlich ausgeprägt sein dürften. Dies wirkt sich in unterschiedlicher Weise auf das aktuelle Sportengagement von älteren Menschen aus. Angesichts der Vielfalt und Bedeutung individueller sportbezogener Sozialisationserfahrungen stellt sich die Frage, (1) wie sich das Sportengagement im lebenszeitlichen Verlauf verändert und (2) welchen Einfluss die sportliche Vorgeschichte auf das Sportengagement in der 2. Lebenshälfte hat. Insbesondere die Bearbeitung der zweiten Fragestellung steht im Mittelpunkt des vorliegenden Beitrags.

\section{Forschungsstand}

\section{Alter und 2. Lebenshälfte}

Alter ist ein schwer zu fassendes soziales Konstrukt, das je nach Kontext unterschiedlich gebraucht und gedeutet wird. Nach Backes und Clemens (2008, S. 11) hat sich „als kulturelles Muster des Eintritts in die ,Lebensphase Alter ${ }^{\prime}(. .$.$) aber$ seit 100 Jahren der Übergang in den $\mathrm{Ru}-$ hestand nach Beendigung des Erwerbslebens herausgebildet“. Diese eher statische Sichtweise wurde jedoch durch eine prozessuale Sichtweise ergänzt. Bei- spielsweise wird im Ansatz der Lebensverlaufsforschung nach Mayer (1990) „Alter als letzte Phase des Durchlaufens der Sozialstruktur" verstanden (Backes \& Clemens, 2008, S. 13). Aus einer soziologischen Perspektive sind die Lebensphase Alter und die 2. Lebenshälfte durch spezifische soziale Rollen und Veränderungen der Identitätsvorstellungen von anderen Lebensphasen zu unterscheiden. Aufgrund der zunehmenden Pluralisierung der Lebensformen erscheint es auf einer inhaltlich-theoretischen Ebene nicht sinnvoll, die Lebensphase Alter und 2. Lebenshälfte durch genaue Angaben zum chronologischen Alter abzugrenzen. Allerdings ist auf empirischer Ebene eine entsprechende Altersfestlegung zur Operationalisierung erforderlich. Aus diesem Grund wird im empirischen Teil dieses Beitrags dem Begriff der 2. Lebenshälfte ein an der durchschnittlichen Lebenserwartung orientiertes Verständnis zugrundegelegt. Sie wird als die Lebensphase verstanden, die ausgehend von der durchschnittlichen Lebenserwartung ungefähr nach dem 40. Lebensjahr beginnt. Das heißt, die 2. Lebenshälfte umfasst das spätere Erwachsenenalter und reicht bis in das mittlere Erwachsenenalter hinein. Auf diese Weise werden zwar die Lebensphase Alter und 2. Lebenshälfte im Vergleich zur üblichen Orientierung am Renteneintritt ausgeweitet, jedoch kann so die Pluralisierung der Lebensformen im Alter auch auf empirischer Ebene berücksichtigt werden. Allerdings ist bei einer solchen Ausdeh- 
nung der 2. Lebenshälfte der soziohistorische Kontext der jeweiligen Alterskohorte zu berücksichtigen.

\section{Zentrale Befunde zum Sportengagement in der 2. Lebenshälfte}

\section{Theoretische Arbeiten}

Die körperlich-sportliche Aktivität von älteren Menschen ist inzwischen Gegenstand nahezu aller sportwissenschaftlicher Teildisziplinen. Die Frage nach den Einflussfaktoren und Determinanten auf die sportliche Aktivität (im Alter) wird von mehreren (sport-)wissenschaftlichen Teildisziplinen behandelt. Insbesondere die Sportpsychologie hat hierzu zahlreiche personale Faktoren und psychologische Konstrukte untersucht, die das Sportengagement im Erwachsenenalter beeinflussen. So spielt beispielsweise das Gesundheitsmotiv vom frühen zum späteren Erwachsenenalter eine immer größere Rolle und wird sehr häufig als Hauptmotiv für den (Wieder-)Beginn einer sportlichen Aktivität genannt. Es ist allerdings eher ein Einsteiger- als ein Dabeibleibermotiv (Pahmeier, 1994, S. 142). Als weitere psychologische Faktoren, welche ein Sportengagement positiv beeinflussen, haben sich beispielsweise die Selbstmotivation sowie die sportbezogene Selbstwirksamkeit erwiesen (u. a. Wagner, 2000, S. 64-69; Woll, 2006, S. 55). Ebenso begünstigt die positive Wahrnehmung des eigenen Gesundheits- und Fitnesszustands die Sportteilnahme (u. a. Pahmeier, 1994, S. 122). Allerdings greift dieser Fokus der psychologischen Perspektive auf ausschließlich personeninterne Faktoren zu kurz, um das komplexe Phänomen einer Sportpartizipation adäquat zu beschreiben oder zu erklären. Eine Ergänzung um soziale Faktoren findet man beispielsweise bei Public-Health-Ansätzen, die zusätzlich soziodemographische und sozioökologische Faktoren wie die Zugänglichkeit zu Sportstätten, das Sportangebot oder die soziale Unterstützung für das Sportengagement berücksichtigen. Sozialisationstheoretische Ansätze umfassen sowohl personale als auch soziale Faktoren (u. a. Hurrelmann, 1998, S. 15-18). Diese allgemeinen sozialisationstheoretischen Ansätze wurden zwar bereits von Brink- hoff (1998) sowie Baur und Burrmann (2000) als theoretische Bezugsrahmen zur sportbezogenen Sozialisation von Jugendlichen zugrundegelegt. Sie wurden jedoch bislang kaum auf die Altersgruppe der Menschen in der 2. Lebenshälfte ausgeweitet bzw. spezifiziert und auch keiner entsprechenden empirischer Überprüfung unterzogen. Neben den bereits erwähnten psychologischen, gesundheitswissenschaftlichen und sozialisationstheoretischen Ansätzen, lieferte bereits Baur (1989) vorwiegend auf theoretischer Ebene umfassende Überlegungen und Erklärungsansätze zu Körper- und Bewegungskarrieren, die zwar die gesamte Lebensspanne umfassen, sich jedoch schwerpunktmäßig auf das Kindes- und Jugendalter beziehen. Aus der Perspektive der Ungleichheitsforschung hat $\mathrm{Na}$ gel (2003, S. 96) in Anlehnung an Lamprecht und Stamm (1995, S. 268-271) ein Mehrebenenmodell zur Analyse sozialer Ungleichheiten im Sport entwickelt, das aber keine Verlaufsperspektive aufzeigt und nur teilweise in der empirischen Studie Berücksichtigung fand. Bei regionalen Studien zum Seniorensport in Brandenburg wurden sowohl Bausteine der Sozialisations- und der Ungleichheitsforschung als auch der Lebenslaufforschung als theoretische Grundlage berücksichtigt, indem insbesondere der Strukturwandel des Alters und das Sportengagement im Kontext von Lebensführung und Lebenslage thematisiert werden (Baur et al., 1996). Insgesamt ist die Zahl an Beiträgen, die sich explizit um eine (theoretisch fundierte) Erklärung des Sportengagements von Menschen in der 2. Lebenshälfte bemühen im Vergleich zu beschreibenden Ansätzen als gering zu bezeichnen.

\section{Empirische Befunde}

Die Mehrzahl der vorliegenden empirischen Studien zur Sportpartizipation beschreibt das Sportengagement zu einem bestimmten Zeitpunkt im Lebensverlauf (mittleres und/oder späteres Erwachsenenalter: Baur et al., 1996; Denk \& Pache, 1996; Hartmann-Tews, Tischer \& Combrink, 2008a; Lamprecht \& Stamm, 2001; Lamprecht, Fischer \& Stamm, 2008; Nagel, 2003; Pache, 2003; Thiel et al., 2008). Der Schwerpunkt liegt hierbei auf der Untersuchung der sozialen Faktoren
Geschlecht, Alter, teilweise Bildung, sozioökonomischer Status sowie Region. Die Befundlage dieser Studien offenbart große Unterschiede zur Verbreitung der Sportaktivität dieser Zielgruppe im mittleren und späteren Erwachsenenalter (Sportbeteiligung zwischen $>\mathbf{2 0} \%$ und $<50 \%)$. Im Vergleich zu jüngeren Alterskohorten ist für Menschen in der 2. Lebenshälfte nach wie vor eine geringere Sportbeteiligung zu konstatieren. Während bei den jüngeren Altersgruppen der Anteil weiblicher Sportaktiven im geschlechtsspezifischen Vergleich geringer ist, kehrt sich das Bild im mittleren Erwachsenenalter um, und es treiben prozentual mehr Frauen als Männer Sport. Zahlreiche Studien belegen darüber hinaus, dass mit zunehmenden Bildungsniveau auch die Sportbeteiligung steigt (u. a. Baur et al., 1996, S. 164-168; Lamprecht \& Stamm, 2001, S. 12; Klein, 2009, S. 21; Nagel, 2003, S. 139-143).

Die verlaufsbezogenen Veränderungen des Sportengagements über die Lebensspanne können nur durch Längsschnittstudien untersucht werden. Die untersuchte Zeitspanne variiert hierbei von wenigen Jahren (2-27 Jahre: Breuer, 2003, 2004; Breuer \& Wicker, 2008, 2009; Becker, Klein \& Schneider, 2006; Eichberg \& Rott, 2004; Jekauc, Woll, Tittlbach \& Bös, 2008; Wagner, 2000; Woll, 2006; Beunen et al., 2004; Kemper, de Vente, van Mechelen \& Twisk, 2001; Kirjonen et al., 2006; Kjønniksen, Anderssen \& Wold, 2009; Kristensen et al., 2008; Scheerder, Vanreusel \& Taks, 2005; Scheerder, Vanreusel, Taks \& Renson, 2002; Tammelin, Näyhä, Hills \& Järvelin, 2003; Telama, Leskinen \& Yang, 1996) bis hin zur Betrachtung der gesamten Lebensspanne (Allmer, 2002; Baur et al., 1996; Breuer \& Wicker, 2007; Becker \& Klein, 2007; Eichberg, 2003; Frändin, Mellström, Sundh \& Grimby, 1995; Frogner, 1991; Klein, 2009; Pitsch, 2005). Die Ergebnisse dieser Längsschnittstudien, die Antworten auf die eingangs formulierte Frage nach den Veränderungen des Sportengagements im lebenszeitlichen Verlauf geben können, widersprechen der aus einer Querschnittsperspektive resultierenden Annahme eines negativen Zusammenhangs zwischen der Sportaktivität und dem Lebensalter (u. a. Breuer, 2003, S. 270; Klein, 2009, S. 14-15). Ab 
dem mittleren Erwachsenenalter bleibt der Anteil der Sportaktiven auf gleichbleibendem Niveau oder steigt sogar leicht an. Der Schwerpunkt der v. a. aus den skandinavischen und den Beneluxländern stammenden wissenschaftlichen Arbeiten liegt auf der Untersuchung des Zusammenhangs zwischen der Sportaktivität im Kindes- bzw. Jugendalter und dem mittleren Erwachsenenalter. Im Gegensatz zu der häufig im Alltag postulierten Annahme eines positiven Zusammenhangs zwischen der Sportaktivität während der Adoleszenz und der Sportpartizipation im Erwachsenenalter bestätigen alle Längsschnittstudien lediglich eine schwache positive Korrelation (Malina, 2001; Beunen et al., 2004; Kuh \& Cooper, 1992; Taylor, Blair, Cummigs, Wun \& Malina, 1999; Trudeau, Laurencelle \& Shephard, 2004; Twisk, Kemper \& van Mechelen, 2000; Telama, Yang, Laakso \& Viikari, 1997). Je länger der betrachtete Zeitraum, desto geringer ist der beobachtete Zusammenhang (Malina, 2001; Beunen et al., 2004; Pfeiffer et al., 2006; McMurray, Harrell, Bangdiwala \& Hu, 200o).

Die Resultate der empirischen Studien sind jedoch nur schwer vergleichbar, da sie sich zum einen auf unterschiedliche Zeiträume beziehen und zum anderen die zentralen Begriffe Sportpartizipation und sportliche Aktivität unterschiedlich definieren und operationalisieren. Ungeachtet dessen ist am derzeitigen Forschungsstand auffallend, dass bislang die Variable Alter vorwiegend als Trägervariable zur differenzierteren Betrachtung des Sportengagements von verschiedenen Altersgruppen berücksichtigt wurde. Die lebenszeitliche Perspektive und die Frage nach lebenszeitlichen Abhängigkeiten werden zwar auf theoretischer und methodischer Ebene zunehmend berücksichtigt, dabei wird aber die sportliche Vorgeschichte kaum theoretisch konzeptualisiert oder auf empirischer Ebene nur ansatzweise erfasst. Bei den vorliegenden Längsschnittstudien wird zwar die Sportpartizipation zu mehreren Messzeitpunkten erhoben, der Verlauf der Sportaktivität dazwischen wird jedoch nicht beachtet. Darüber hinaus findet weder eine Einbettung in die jeweilige Lebensphase mit ihren spezifischen Entwicklungsaufgaben und sozialen Rollen in verschiede- nen Lebensbereichen statt noch eine Einordnung in die historische Zeit, welche die Erfahrungsmöglichkeiten und Bedingungen des Lebenslaufs einzelner Altersgruppen und Geburtskohorten ganz spezifisch prägt.

Angesichts der defizitären Forschungslage soll im Weiteren ein theoretisch-methodischer Ansatz erarbeitet werden, der durch die Analyse der gesamten Lebensspanne zum einen die Untersuchung lebenszeitlicher Abhängigkeiten des Sportengagements und zum anderen die Analyse des Einflusses verschiedener Lebensbereiche sowie der historischen Zeit auf den lebenszeitlichen Verlauf des Sportengagements ermöglicht.

\section{Theoretischer Bezugsrahmen}

Als theoretischer Rahmen wird der Ansatz der Lebensverlaufsforschung gewählt (Elder, 1996; Mayer, 1990; in der Sportwissenschaft Baur, 1998), der individuelle Lebensverläufe und gesamtgesellschaftliche Prozesse in einem einheitlichen „formalen, kategorialen und empirischen Bezugsrahmen" (Mayer, 1990, S. 11) betrachtet ${ }^{1}$. Vor diesem Hintergrund werden individuelle Lebensverläufe als Karrieren bezeichnet, die als Abfolge von Aktivitäten und Ereignissen in verschiedenen Lebensbereichen sowie in den jeweiligen Institutionen und Organisationen zu betrachten sind. „Der Lebensverlauf ist ein endogener Kausalzusammenhang" (Mayer, 1990, S. 11), d. h. die einzelnen Karrieren in den jeweiligen Lebensbereichen sind nicht situativ und isoliert zu verstehen, sondern aus den Bedingungen der vorausgegangenen Lebensgeschichte zu erklären. Einzelne Lebensphasen und -abschnitte, z. B. Kindheit, Jugend, Erwachsenenalter und Alter, müssen im Zusammenhang betrachtet werden. Des Weiteren sind die Verläufe in den anderen Lebensbereichen zu beachten. Da die Lebensverläufe von der Abfolge der Geburtskohorten bestimmt werden und zumindest teilweise insti-

\footnotetext{
1 Aufbauend auf dem Ansatz der Lebensverlaufsforschung haben Nagel und Conzelmann (2006) ein spezifisches Konzept zur Analyse von Sportkarrieren von Hochleistungssportlern entwickelt (vgl. auch Conzelmann, 2001; Nagel, 2002).
}

tutionalisiert sind, sind sie von individuellen Biographien zu unterscheiden. Altersnormen und kulturell vermittelte Vorstellungen können hierbei als „sozialisatorische Verstärker“ dienen (Mayer, 1990, S. 10).

Gegenstand der Lebensverlaufsforschung sind sowohl institutionell definierte Ereignisse (z. B. der Eintritt in einen Sportverein) als auch die relative Verweildauer in bestimmten Aktivitäten, beispielsweise die Dauer des Sportengagements. Demzufolge konstituiert das im Lebensverlauf sich verändernde Sportengagement die Sportkarriere. Als Teil des Freizeitbereichs ist die Sportkarriere zwar im Vergleich zu anderen Teilverläufen relativ frei individuell gestaltbar, sie ist jedoch gleichwohl auch von institutionellen und organisatorischen Rahmenbedingungen abhängig. Wer sich einem Leistungsvergleich im Wettkampf stellen möchte, muss sich nach wie vor in vielen Sportarten einem Verein anschließen, um am Wettkampfangebot der Verbände partizipieren zu können.

Das aktuelle Sportengagement steht in Zusammenhang mit der sportlichen Vorgeschichte und ist nur mit Blick auf die sportlichen Vorerfahrungen zu verstehen. Im Verlauf der Sportkarriere „erwirbt die Person dabei individuell-subjektive Erfahrungen in Bezug auf Körper und Bewegung, die dann ihr künftiges Handeln wiederum leiten“" (Baur, 1989, S. 86). Individuell unterschiedliche sportliche Erfahrungen im Kindes- und Jugendalter werden sich auf das Sportengagement im weiteren Lebensverlauf vermutlich spezifisch auswirken. Positive Erfahrungen des sozialen Zusammenhalts in einer Sportmannschaft beispielsweise könnten dazu führen, dass auch im weiteren Lebensverlauf eine Sportaktivität in der Gruppe dem Sporttreiben alleine vorgezogen wird. Dagegen könnten negative Erfahrungen, beispielsweise ein zu hoher Leistungsdruck im Jugendalter, zu einem Abbruch des Sportengagements führen, und erst im höheren Erwachsenenalter wird der sportliche Wiedereinstieg gefunden. Über die gesamte Lebensspanne weist das Sportengagement einen dynamischen Verlauf auf, d. h. auf Phasen intensiven Sportengagements können Phasen geringer bzw. un- 
regelmäßiger Sportbeteiligung und Phasen der Sportabstinenz folgen. Der dynamische Verlauf der Sportkarriere bezieht sich darüber hinaus auf mögliche Sportartenwechsel sowie auf die Veränderungen weiterer Aspekte des Sportengagements, z. B. Häufigkeit, Wettkampforientierung oder Organisationsform.

Bei der Analyse der individuellen Sportkarriere sind die Verläufe in anderen Lebensbereichen zu berücksichtigen. Denn die Sportkarriere kann durch Ereignisse der familialen und beruflichen Teilverläufe beeinflusst werden: wie etwa durch hohe zeitliche Belastungen in beruflichen Etablierungsphasen oder die Geburt von Kindern, aber auch im späteren Erwachsenenalter durch den Beginn des Ruhestands oder die Pflege eines Angehörigen.

Die Sportkarriere steht weiterhin unter dem Einfluss sich verändernder gesellschaftlicher Rahmenbedingungen. Es ist anzunehmen, dass sich verschiedene Alterskohorten nicht nur in Bezug auf ihr Sportverständnis, sondern auch in Bezug auf ihre individuellen sportlichen Vorgeschichten unterscheiden: „Die heute Alten kommen teilweise aus einer Generation, in der zeitlebens weniger Sport betrieben wurde“ (Klein, 2009, S. 7). Alterskohorten, die ihre Kindheit und Jugend während und kurz nach dem Zweiten Weltkrieg erlebten, werden durch den erschwerten Zugang zum Sport in dieser Lebensphase wahrscheinlich ein geringeres Sportengagement aufweisen (vgl. Baur et al., 1996, S. 26-28). Noch bis Mitte des 20. Jahrhunderts war ein aus heutiger Sicht enges Sportverständnis vorherrschend und Sport war überwiegend eine Freizeitbeschäftigung von jungen Männern aus höheren sozialen Schichten. Sowohl Mädchen und junge Frauen als auch Ältere waren vergleichsweise weniger häufig sportaktiv. Auch hatte die Freizeit noch nicht den heutigen Stellenwert. Erst durch die Verkürzung der Wochenarbeitszeit sowie die Öffnung und Ausdifferenzierung des vormals ausschließlich leistungs- und wettkampforientierten Sports haben weitere Personengruppen den Zugang zum Sport gefunden. Ein zunehmendes Gesundheitsbewusstsein sowie die Orientierung an Körper- und Jugendlich-

\section{Sportwiss $2011 \cdot 41: 216-232$ DOI 10.1007/s12662-011-0198-4}

C Springer-Verlag 2011

\section{Claudia Klostermann · Siegfried Nagel}

\section{Sport treiben ein Leben lang? Einfluss der Sportkarriere der 1. Lebenshälfte auf das Sportengagement im mittleren und späten Erwachsenenleben}

\section{Zusammenfassung}

Aktuelle demographische Entwicklungen rücken Fragen nach den Einflussfaktoren der Sportbeteiligung von Menschen in der 2. Lebenshälfte in den Mittelpunkt sportwissenschaftlichen Interesses. Aufgrund der vielfältigen Lebenserfahrungen dieser Altersgruppe stellt sich die Frage, inwieweit die sportliche Vorgeschichte das aktuelle Sportengagement beeinflusst. Ausgehend vom Ansatz der Lebensverlaufsforschung wurden hierzu Personen ab dem 50. Lebensjahr zu ihrem aktuellen und früheren Sportengagement im retrospektiven Längsschnitt befragt. Die Ergebnisse zeigen, dass insbesondere ein langjähriges Sportengagement in der 1. Lebenshälf- te sowie sportliche Aktivitäten im frühen Erwachsenenalter den Verlauf des Sportengagements in der 2. Lebenshälfte positiv beeinflussen. Darüber hinaus weisen Periodenund Kohorteneffekte darauf hin, dass die lebenszeitlichen Abhängigkeiten des Sportengagements unter dem moderierenden Einfluss des sozialen Faktors Geschlecht sowie gesellschaftlicher Rahmenbedingungen stehen.

\section{Schlüsselwörter}

Sportpartizipation · Sportkarrieren .

Lebensverlauf · Erwachsenenalter

\section{Sports for life? The influence of sport careers in the first half of life on sport participation among older people}

\section{Abstract}

Current demographic development attracts the attention of the sports sciences to the determinants of sport participation among people in the second half of life. Due to various life experiences in this age cohort, the question arises as to how much sports experiences affect contemporary sport participation. Starting with research into the life course people had followed, persons aged over 50 years were interviewed retrospectively about their current and former participation in sporting activities. Results show that sport participation of many years' standing in the first half of life in particular, as well as sport activities in young adulthood, has a positive affect on the course of sport participation in the second half of life. Furthermore, period and cohort effects indicate that sports commitment is moderately affected by the variables sex and social environment.

\section{Keywords}

Sport participation · Sport careers · Life course $\cdot$ Adulthood 
keitsidealen haben in Verbindung mit sportpolitischen Programmen zur Bewegungsförderung eine Zunahme der Sportpartizipation bewirkt. In den vergangenen Jahrzehnten ist v. a. die Sportbeteiligung der Frauen stetig angestiegen, so dass heute die Sportbeteiligung bei Frauen im mittleren Erwachsenenalter ungefähr derjenigen der gleichaltrigen Männer entspricht (Breuer, 2003, S. 271-272; Breuer, 2004, S. 53-54; Klein, 2009, S. 14). Es ist anzunehmen, dass diese Sportentwicklung unterschiedlichen Einfluss auf die Sportaktivität von verschiedenen Alterskohorten hatte. Ältere Alterskohorten werden auf vergleichsweise wenig Sporterfahrung zurückgreifen können. Durch die gesellschaftliche Bedeutungszunahme des Sports und die Betonung der positiven Wirkungen auf die Gesundheit aller Altersgruppen allerdings sehen die Jüngeren wie die Älteren heute Sport und Bewegung als ein „positiv konnotiertes und normativ verankertes Medium, um sich jung, fit und gesund zu halten" (Hartmann-Tews, Tischer \& Combrink, 2008b, S. 47).

In Deutschland ist darüber hinaus die besondere Situation gegeben, dass es bis zur Wiedervereinigung 1990 zwei verschiedene Sportsysteme gab, welche die individuellen Sportkarrieren spezifisch beeinflussten. Der Schwerpunkt des ostdeutschen Sportsystems lag aus ideologischen und politischen Gründen auf der Förderung des Spitzensports. Die Massensportbewegung wurde politisch konstruiert und zentralistisch mit dem Ziel der Erziehung zum sozialistischen Menschen installiert (u. a. Baur et al., 1996, S. 110; Hinsching, 1998, S. 17; Trillitzsch, 2004, S. 94-96). Im Gegensatz dazu konnte sich der Freizeit- und Breitensport in Westdeutschland aufbauend auf einem eigenständigen Sportsystem, das föderalistisch und nach dem Subsidiaritätsprinzip organisiert ist, unabhängig von politischer Einflussnahme entwickeln. Nach der politischen Wiedervereinigung im Jahr 1990 wurde der Transformationsprozess des ostdeutschen Sportsystems in ein gesamtdeutsches Sportsystem durch einen raschen organisatorischen Zusammenschluss auf Bundes-, Landes- und Kommunalebene angestoßen. Die sporttreibenden Men- schen in Ostdeutschland sahen sich nach dem politischen Umsturz mit einem völlig anders organisierten Sportsystem konfrontiert. Vor dem Hintergrund dieser Ausführungen ist deshalb zu vermuten, dass sich die unterschiedlichen Rahmenbedingungen der beiden Sportsysteme auf die individuellen Sportkarrieren auswirkten.

Ausgehend von der eingangs formulierten Problemstellung wird im Rahmen des vorliegenden Beitrages der Fokus auf die Sportkarriere gerichtet. Dabei werden die Karrieren in anderen Lebensbereichen ausgeblendet und der Einfluss sich verändernder gesellschaftlicher Rahmenbedingungen (kohortenspezifische Einflüsse, unterschiedliche Sportsysteme in Ost- und Westdeutschland) im Sinne von moderierenden Variablen berücksichtigt. Im Folgenden werden die lebenszeitlichen Abhängigkeiten der Sportkarriere aus einer sozialisationstheoretischen Perspektive präzisiert (Baur, 1989):

1. Zunächst scheint von Bedeutung zu sein, in welchen Lebensphasen Sport getrieben wird. Hierbei ist zu vermuten, dass ein Sportengagement insbesondere im Kindes- und Jugendalter im Sinne einer frühzeitigen Sozialisation zum Sport eine zentrale Rolle spielt. Wer frühzeitig sportliche Bewegungsformen und Sportarten erlernt und sportive Einstellungen erwirbt, wird vermutlich eher lebenslang Sport treiben. In diesem Zusammenhang ist auch zu beachten, inwieweit das Sportengagement nach der Jugendphase dauerhaft weitergeführt wird. Insgesamt dürften sich langjährige sportliche Vorerfahrungen in der 1. Lebenshälfte eher förderlich für regelmäßige Sportaktivitäten in der 2. Lebenshälfte auswirken. Nur durch ein kontinuierliches Sportengagement über mehrere Jahre hinweg wird das Sportreiben zur Gewohnheit, zum festen Bestandteil der Lebensführung und Teil des Lebensstils.

2. Weiterhin sind die Regelmäßigkeit und die organisatorische Einbindung des Sportengagements zu berücksichtigen. Dabei dürfte in Sportvereinen aufgrund des institutionalisierten Trainingsbetriebs eine besonders intensive Auseinandersetzung mit spor- tiven Inhalten erfolgen. Vor allem im Leistungs- und Wettkampfsport können Kinder, Jugendliche und junge Erwachsene besondere Erfahrungen machen, die möglicherweise für das Sportengagement in der 2. Lebenshälfte von Bedeutung sind.

3. Die individuellen Sportkarrieren sind zudem darin zu unterscheiden, ob sie eher durch ein vielseitiges Sportengagement oder durch die Spezialisierung auf eine Sportart geprägt sind. Durch eine vielseitige Sportkarriere, in deren Verlauf verschiedene Sportarten in unterschiedlichen Organisationsformen (Verein, selbstorganisiert, kommerzielle Sportanbieter usw.) ausgeübt werden, können Erfahrungen gesammelt und Kompetenzen erworben werden, auf die im weiteren Verlauf der Sportkarriere zurückgegriffen werden kann. Dagegen erschließen sich durch die Spezialisierung in einer Sportart andere Erfahrungen und Möglichkeiten. Entscheidet man sich für die leistungssportliche Karriere, so wird man sich ab einem gewissen Leistungsniveau auf eine Sportart konzentrieren müssen.

Im Zusammenhang mit den postulierten Vermutungen interessiert weiterhin der Einfluss der Variablen Geschlecht, Alterskohorte, Wohnort und Bildungsabschluss? ${ }^{2}$ Zum Verlauf des Sportengagements in der Lebensspanne sollen folgende Fragestellungen empirisch untersucht werden:

- Inwiefern unterscheiden sich die individuellen Sportkarrieren von Männern und Frauen sowie von verschiedenen Alterskohorten?

- Inwiefern unterscheiden sich die individuellen Sportkarrieren von Menschen in West- und Ostdeutschland?

- Inwiefern unterscheiden sich die individuellen Sportkarrieren in Abhängigkeit vom Bildungsniveau?

\footnotetext{
2 Hierbei könnten weitere Einflussvariablen (z. B. Haushaltskonstellation, Einkommen) untersucht werden. In der vorliegenden empirischen Studie "Sport im Lebenslauf" konnten jedoch aus forschungspragmatischen Gründen nur die oben genannten Variablen berücksichtigt werden.
} 


\section{Methode}

\section{Untersuchungsdesign}

Zur Rekonstruktion der Sportkarrieren von Menschen in der 2. Lebenshälfte wurde im Jahr 2008 eine retrospektive Längsschnittstudie durchgeführt. Insgesamt wurden 1739 Personen ab dem 50. Lebensjahr in Chemnitz ( $n=881$; Zufallsstichprobe) und Braunschweig ( $n=858$; Zufallsstichprobe) telefonisch zu ihrem Sportengagement befragt. Zur Berücksichtigung des Einflusses der unterschiedlichen Sportentwicklungen in West- und Ostdeutschland auf die individuellen Sportkarrieren wurden zwei in Bezug auf die Bevölkerungsstruktur (z. B. Einwohnerzahl, Anteil der über 50-Jährigen an der Gesamtbevölkerung, Arbeitslosenquote) vergleichbare Städte in Ost- und Westdeutschland gewählt (theoretische Auswahl). Die Zufallsstichproben wurden anhand des Random-Last-Digit-Verfahrens gezogen (vgl. Schnell, Hill \& Esser, 2008, S. 292). Um die Sportkarriere in der 2. Lebenshälfte im lebenszeitlichen Verlauf beleuchten zu können, wurde als Mindestalter das 50. Lebensjahr gewählt. Zur Erfassung von möglichen zeithistorischen Effekten wurde ein kohortenanalytisches Design gewählt, und es wurden Personen der Geburtsjahrgänge 1929 bis 1958 befragt. Zur Erfassung des Sportengagements in der Lebensspanne wurde ein Fragebogen entwickelt, der sich an bereits bestehenden Fragebögen orientiert. ${ }^{3}$

\section{Methodische Probleme retrospektiv erhobener Daten}

Da retrospektiv erhobene Daten mit dem methodischen Problem der mangelnden Erinnerungsfähigkeit und der Tendenz zur biografischen Glättung der Befragten konfrontiert sind, wurden dem theo-

\footnotetext{
${ }^{3}$ Mit Ausnahme der Studie von Pitsch (2005) zur Sportaktivität der saarländischen Bevölkerung und der aktuellen Studie „Ein aktives Leben leben - Alter und Altern in Baden-Württemberg" (Klein, 2009) wurden bislang die Sportkarrieren entweder nichtdifferenziert nach Sportarten (u. a. Baur et al., 1996) oder nur von Hochleistungssportler(inne)n (u. a. Côté, Ericsson \& Law, 2005; Nagel \& Conzelmann, 2006) retrospektiv erfasst.
}

retisch-methodischen Ansatz der Lebensverlaufsforschung folgend nur objektive Elemente des Sportengagements im Lebensverlauf (Sportart, Häufigkeit, Wettkampforientierung usw.) erfasst, anstatt subjektiver Deutungen persönlicher Erlebnisse im Sportengagement oder auch subjektiver Einschätzungen zur Intensität (Ropponen, Levälahti, Simonen, Videman \& Battié, 2001). Die Abfolge der Ereignisse im Sportengagement wird durch die Abfolge der häufigsten Sportarten abgebildet. Trotz der zeitlichen Strukturierung bildet das Ereignis der Ausübung einer Sportart den Ausgangspunkt, welches dann in den zeitlichen Verlauf eingeordnet wird (Brückner, 1990, S. 382). Zunächst wurden alle in der jeweiligen Lebensdekade betriebenen Sportarten aufgelistet (vgl. Côté, Ericsson \& Law, 2005, S. 4-5). Danach wurde die häufigste Sportart herausgestellt, die durch weitere Fragen (Häufigkeit, Wettkampforientierung) spezifiziert und schließlich durch die Angabe von Beginn und Ende ihrer Ausübung in die Zeitachse eingeordnet wurde. Nach Klein (2009, S. 9) ist mit der „Differenzierung zwischen den Sportarten eine höhere Datenqualität verbunden als in Untersuchungen, in denen nur generell nach sportlicher Aktivität gefragt wird“. In der vorliegenden Studie wurde die Sportaktivität über die regelmäßige Ausübung der Sportarten außerhalb des Schulsports, d. h. mindestens ein halbes Jahr lang und mindestens einmal pro Woche, operationalisiert. Mit dieser eher engen Definition kann ein zuverlässigeres Antwortverhalten gewährleistet werden, da die Erinnerungsfähigkeit sowohl von der Dauer der Aufrechterhaltung der Sportaktivität als auch von der subjektiven Bedeutung des Ereignisses abhängig ist (u. a. Reimer \& Matthes, 2007, S. 4).

Zur Reliabilitätsüberprüfung wurde eine ergänzende Studie durchgeführt, bei der analog der Test-Retest-Methode insgesamt 38 Personen zu 2 Messzeitpunkten mittels des entwickelten Fragebogens zu ihrem aktuellen und früheren Sportengagement telefonisch befragt wurden. Der Zeitraum zwischen der 1. und zweiten Erhebung betrug etwa 5 Monate. $\mathrm{Zu}$ beiden Erhebungszeitpunkten nahmen 18 Frauen und 20 Männer im Alter von 64 bis 88 Jahren teil.
Berechnet wurde die Reliabilität je nach Skalenniveau entweder deskriptiv über die prozentuale Übereinstimmung der Angaben oder durch die Korrelationsmaße Cramers Index (CI), dem Produkt-Moment-Korrelationskoeffizienten nach Pearson bei Normalverteilung ( $r$ ) bzw. dem Rangkorrelationskoeffizienten nach Spearman $\left(\mathrm{r}_{\mathrm{s}}\right)$ bei nicht normalverteilten Variablen ( $\mathbf{\bullet}$ Tab. 1).

Insgesamt wurden zu beiden Erhebungszeitpunkten überwiegend die gleichen Angaben gemacht (• Tab. 1). Die mittels des Fragebogens erhobenen retrospektiven Daten weisen eine überwiegend hohe Stabilität (Bortz \& Döring, 2006, S. 196) auf. Insbesondere die Angaben, ob in der jeweiligen Lebensdekade Sport getrieben wurde oder nicht, stimmen zu beiden Erhebungszeitpunkten weitestgehend überein. Die Angaben zur häufigsten Sportart in der jeweiligen Lebensdekade weisen dagegen eine etwas geringere Stabilität auf, insbesondere in der 3. Lebensdekade (20.-29. Lebensjahr). Stimmen dagegen zu beiden Erhebungszeitpunkten die Angaben zur häufigsten Sportart in der jeweiligen Lebensdekade überein, so ist die Erinnerung an das Lebensalter bei Beginn und Ende dieser Sportart wieder sehr stabil. Auch die Erinnerungen in die am weitesten zurückliegende Kindheit weisen eine sehr hohe Stabilität auf.

Allerdings lässt sich durch die gewählte Test-Retest-Methode keine Aussage über die Richtigkeit der Angaben machen. Aus diesem Grund erfolgte vor der Datenauswertung eine umfangreiche und sorgfältige Datenbereinigung und Datenedition durch inhaltliche Quervergleiche und interne Konsistenzprüfungen (z. B. durch einen Vergleich der Angaben zur Wettkampfteilnahme zur Häufigkeit der Ausübung der Sportart). Dennoch ist es nicht gänzlich auszuschließen, dass bei der gewählten Erhebungsform in der Erinnerung Zeitraffer- und Zeitlupeneffekte auftreten können (Reimer \& Matthes, 2007, S. 712). Hierbei handelt es sich jedoch vermutlich um keinen systematischen (Mess-)Fehler.

Insgesamt kann also davon ausgegangen werden, dass mit Hilfe des entwickelten Erhebungsinstruments trotz des gegebenen Problems der Erinnerungsfähigkeit zuverlässige Daten zum Sportengagement 


\begin{tabular}{|c|c|c|c|c|}
\hline & $\begin{array}{l}\text { Sportaktivi- } \\
\text { tät (ja/nein) }\end{array}$ & $\begin{array}{l}\text { Hauptsportart (häu- } \\
\text { figste Sportart) }\end{array}$ & $\begin{array}{l}\text { Alter bei Beginn } \\
\text { der Hauptsportart }\end{array}$ & $\begin{array}{l}\text { Alter bei Ende der } \\
\text { Hauptsportart }\end{array}$ \\
\hline & $\begin{array}{l}\text { Korrelations- } \\
\mathrm{maß}\end{array}$ & $\begin{array}{l}\text { \% der Übereinstim- } \\
\text { mung }\end{array}$ & Korrelationsmaß & Korrelationsmaß \\
\hline 1. Lebensdekade & $\mathrm{Cl}=1,00$ & 91,7 & $r=0,839$ & $r=0,997$ \\
\hline 2. Lebensdekade & $\mathrm{Cl}=0,947$ & 87,0 & $r=0,709$ & $r_{s}=0,979$ \\
\hline 3. Lebensdekade & $\mathrm{Cl}=0,836$ & 70,4 & $r=0,920$ & $r=0,989$ \\
\hline 4. Lebensdekade & $\mathrm{Cl}=0,823$ & 80,8 & $r=0,943$ & $r=0,993$ \\
\hline 5. Lebensdekade & $\mathrm{Cl}=1,00$ & 89,3 & $r=0,923$ & $r=0,929$ \\
\hline $\begin{array}{l}\text { Aktuelles Sport- } \\
\text { engagement }\end{array}$ & $\mathrm{Cl}=1,00$ & 84,2 & $r=0,954$ & - \\
\hline
\end{tabular}

Tab. 2 Stichprobenbeschreibung:Wohnort differenziert nach Geschlecht, Alter, Bildungsabschluss und aktueller Sportaktivität $(n=1739)$

\begin{tabular}{|c|c|c|c|c|c|}
\hline \multirow{3}{*}{ Gesamt } & & \multicolumn{2}{|c|}{ Chemnitz } & \multicolumn{2}{|c|}{ Braunschweig } \\
\hline & & $\mathbf{n}$ & $\%$ & $\mathbf{n}$ & $\%$ \\
\hline & & 881 & 50,7 & 858 & 49,3 \\
\hline \multirow[t]{2}{*}{ Geschlecht } & Weiblich & 539 & 61,2 & 524 & 61,1 \\
\hline & Männlich & 342 & 38,8 & 334 & 38,9 \\
\hline \multirow[t]{3}{*}{ Alterskohorte } & 50-59 Jahre (Jhg. 1958-1949) & 286 & 32,5 & 296 & 34,5 \\
\hline & 60-69 Jahre (Jhg. 1948-1939) & 353 & 40,1 & 278 & 32,4 \\
\hline & 70-79 Jahre (Jhg. 1938-1929) & 242 & 27,5 & 284 & 33,1 \\
\hline \multirow{4}{*}{$\begin{array}{l}\text { Bildungsab- } \\
\text { schluss }\end{array}$} & Kein Abschluss & 27 & 3,1 & 85 & 9,9 \\
\hline & $\begin{array}{l}\text { Betriebliche bzw. schulische Berufsausbildung } \\
\text { (Lehre/Berufsschule) }\end{array}$ & 455 & 51,6 & 505 & 58,9 \\
\hline & Fachhochschul- bzw. Hochschulabschluss & 397 & 45,1 & 256 & 29,8 \\
\hline & Anderer (ausländischer) Bildungsabschluss & 2 & 0,2 & 12 & 1,4 \\
\hline \multirow{2}{*}{$\begin{array}{l}\text { Sportaktivität } \\
\text { aktuell }\end{array}$} & Nein & 321 & 36,4 & 326 & 38,0 \\
\hline & $\mathrm{Ja}$ & 560 & 63,6 & 532 & 62,0 \\
\hline \multirow{4}{*}{$\begin{array}{l}\text { Charakteri- } \\
\text { sierung der } \\
\text { aktuellen } \\
\text { Sportaktivität }\end{array}$} & Bewegungsaktive Erholung & 222 & 39,6 & 149 & 28,2 \\
\hline & Gesundheitsorientierte Sportaktivität & 226 & 40,4 & 252 & 47,7 \\
\hline & Sport treiben & 110 & 19,6 & 118 & 22,3 \\
\hline & Leistungssport & 2 & 0,4 & 9 & 1,7 \\
\hline
\end{tabular}

über die gesamte Lebensspanne erhoben werden können.

\section{Stichprobe}

Differenziert man die Stichprobe $(\mathrm{n}=1739)$ nach dem Alter, so zeigt sich, dass etwa je ein Drittel der Befragten bis 59 Jahre alt $(33,5 \%)$, zwischen 60 und 69 Jahre alt $(36,3 \%)$ und zwischen 70 und 79 Jahre $(30,2 \%)$ alt ist. Insgesamt haben mehr Frauen $(61,1 \%)$ als Männer an der Befragung teilgenommen, wobei aufgrund der längeren Lebenserwartung der Anteil der Frauen in der Alterskohorte der 70- bis 79-Jährigen mit 63,3\% erwartungsgemäß etwas größer ist. Die beiden Teilstichproben Chemnitz und Braunschweig unterscheiden sich in Bezug auf das Bildungsniveau der befragten Personen.

\section{Ergebnisse}

\section{Sportaktivität im Lebensverlauf}

Der Verlauf der Sportaktivität über die Lebensspanne wird in $\bullet$ Abb. 1 differenziert nach Geschlecht beschrieben. Der Anteil der Sportaktiven im Lebensverlauf ist im Jugendalter mit rund $61 \%$ am größten. Er sinkt im jungen und mittleren Erwachsenenalter (4. Lebensdekade) auf ca. $40 \%$ und steigt bis zur 7 . Lebensdekade auf $56,4 \%$ kontinuierlich an (Breuer, 2003, S. 270; Klein, 2009, S. 14).

Dabei sind für die jüngeren Lebensdekaden geschlechtsspezifische Unterschiede festzustellen. Ab der 4. Lebensdekade jedoch steigt der Anteil der Sportaktiven unter den Frauen im Vergleich zu den Männern stärker an und ungefähr $\mathrm{ab}$ der 5. Lebensdekade weisen die Sportbeteiligungsquoten keine geschlechtsspezifischen Unterschiede mehr auf (Breuer, 2003, S. 271-272; Breuer, 2004, S. 53-54; Klein, 2009, S. 14).

Angesichts der bereits diskutierten methodischen Probleme der retrospektiven Datenerfassung stellt sich die Frage, ob der Zuwachs der Sportbeteiligung im mittleren und späteren Erwachsenenalter darauf zurückzuführen ist, dass weniger lang zurückliegende Aktivitäten und Ereignisse besser erinnert werden als länger zurückliegende. Dieser Vermutung widerspricht jedoch, dass die prospektiv ermittelten Paneldaten von Breuer (2003, 2004) ähnliche Befunde liefern. Allerdings könnte die Zunahme der Sportbeteiligung in der 2. Lebenshälfte auch auf ein verändertes Sportverständnis zurückzuführen sein. Die Selbsteinschätzung der aktuellen Sportaktivität verweist jedoch auf ein breites Sportverständnis der Befragten: Von den aktuell sportlich Aktiven charakterisieren etwas mehr als ein Drittel $(34,8 \%)$ ihre aktuelle Sportaktivität als „,bewegungsaktive Erholung“, 43,6\% als "gesundheitsorientierte Sportaktivität“, ein Fünftel (20,6\%) als „Sport treiben“ und nur 0,9\% als „Leistungssport" (- Tab. 2). Da bei der Befragung das aktuelle Sportengagement vor den Sportaktivitäten in früheren Lebensphasen erfragt wurde, ist es wahrscheinlich, dass dieses aktuelle Sportverständnis auch den Erinnerungen zugrundegelegt wurde und 
so retrospektiv Bewegungsaktivitäten als Sport definiert wurden, die in der jeweiligen Lebensphase nicht als Sporttreiben interpretiert worden wären. Zum Beispiel ist zu vermuten, dass das Fahrrad in der Kindheit und Jugendzeit zur jeweiligen historischen Zeit eher als Fortbewegungsmittel für den Schul- bzw. Arbeitsweg denn als Sportgerät verstanden worden wäre. Bei der vorliegenden Studie gaben jedoch einige Befragten aufgrund des heutigen breiten Sportverständnisses Radfahren als häufigste Sportart in dieser Lebensphase an.

Die differenzierte Betrachtung der Alterskohorten verdeutlicht ( $\bullet$ Abb. 2), dass die Sportaktivität über alle Alterskohorten hinweg einen ähnlichen lebenszeitlichen Verlauf aufweist.

Unter den beiden ältesten Alterskohorten der 70- bis 79-Jährigen und 60bis 69-Jährigen, die ihre Kindheit während des Zweiten Weltkriegs bzw. in den Nachkriegsjahren verbrachten, fand nur jeder Fünfte im Kindesalter den Zugang zum Sport. Erst im Jugendalter stieg ihre Sportbeteiligung an. Demgegenüber war die jüngste Alterskohorte der 50- bis 59-Jährigen bereits im Kindes- und Jugendalter häufiger sportlich aktiv. Einen Hinweis auf einen Alterseffekt stellt die von der historischen Zeit unabhängige, relativ hohe Drop-Out-Quote von ca. 15-20\% von der Jugendzeit zum jungen Erwachsenalter (3. Lebensdekade) in allen 3 Alterskohorten dar. In den 198oer und 1990er Jahren unterscheidet sich die Sportbeteiligung der 3 Alterskohorten kaum mehr voneinander. Aufgrund der zunehmenden Bedeutung und Ausdifferenzierung des Sports in der Gesellschaft in den vergangenen Jahren dürfte die Zunahme der Sportbeteiligung in den vergangenen 10 Jahren als Periodeneffekt zu deuten sein. Bei der Differenzierung nach dem Wohnort werden Unterschiede im Verlauf der Sportkarriere insbesondere bei der jüngsten Alterskohorte der 59bis 6o-Jährigen deutlich. Im Vergleich zu Braunschweig weist diese Alterskohorte in Chemnitz in der Jugendzeit eine höhere Sportbeteiligung auf. Sie sinkt jedoch im Übergang zur 3. Lebensdekade weit mehr als in Braunschweig. Im ostdeutschen Sportsystem wurde insbesondere in den 1970er und 1980er Jahren der leis-

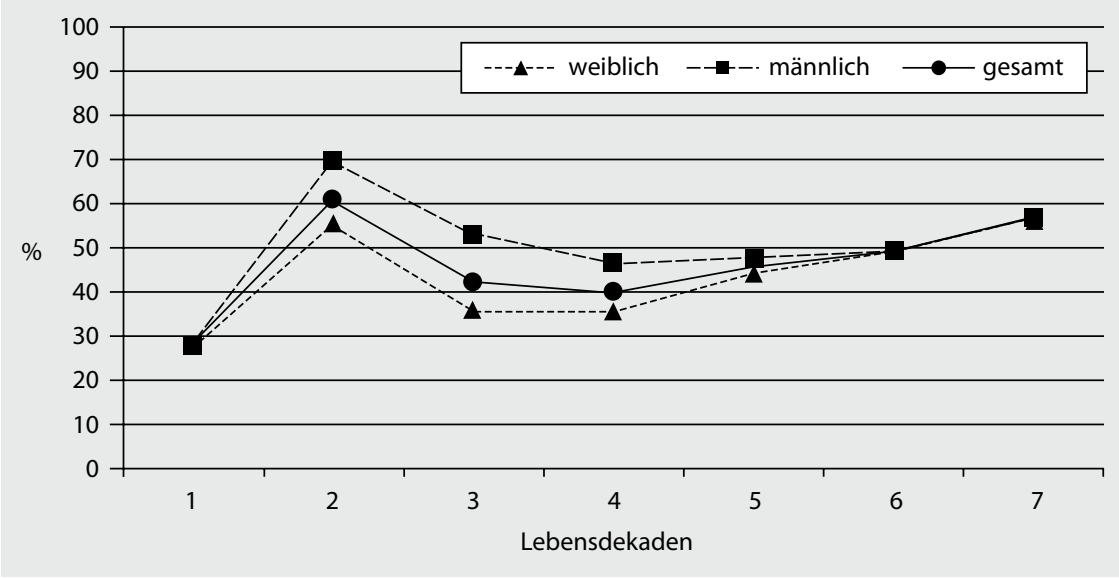

Abb. 1 \ Anteil der Sportaktiven im Lebenslauf differenziert nach Geschlecht (1.-5. Lebensdekade: $n=1739 ; 6$. Lebensdekade: $n=1668 ; 7$. Lebensdekade: $n=1118$ )

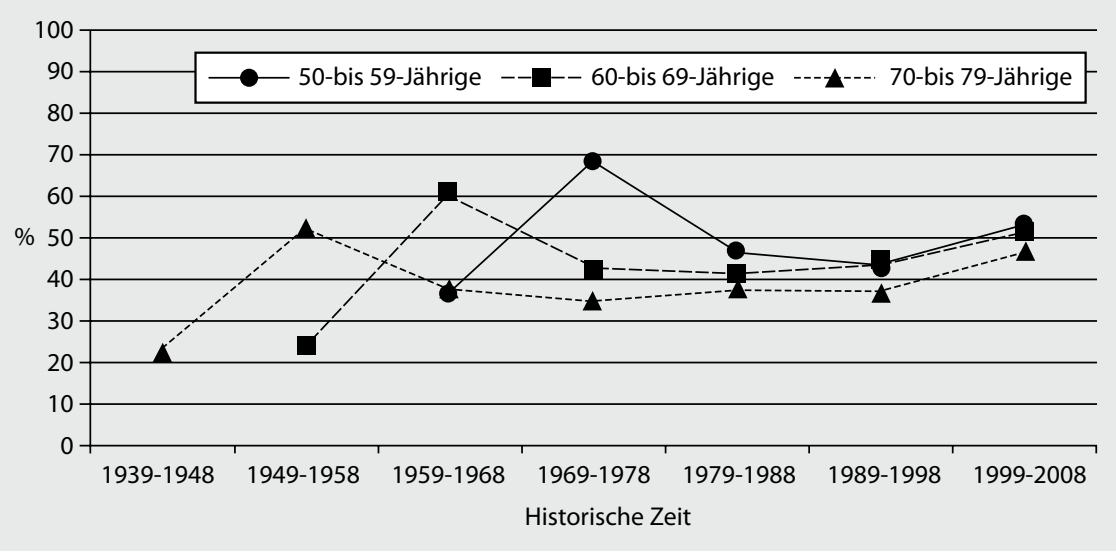

Abb. $2 \Delta$ Anteil der Sportaktiven im Lebenslauf differenziert nach Alterskohorten ( $n=1739)$

tungs- und wettkampforientierte Sport im Kindes- und Jugendalter gefördert, wenngleich es auch sportpolitische Bemühungen gab, einen breitensportorientierten „Freizeit- und Erholungssport“ für breite Bevölkerungsmassen zu etablieren (vgl. Hinsching, 1998, S. 16-20). Dieser Freizeit- und Erholungssport war jedoch ebenfalls sehr wettkampf- und leistungsorientiert und "nach westdeutscher Lesart handelte es sich um einen Leistungssport auf mittleren und unteren Wettkampfebenen“ (Baur et al., 1996, S. 106). Zwar entwickelten sich auch einige breiten- und gesundheitssportliche Sportangebote wie beispielsweise die Ausgleichs- oder Popgymnastik, nicht jedoch ein so differenziertes und vielfältiges Breiten- und Gesundheitssportangebot wie in Westdeutschland. Vermutlich fiel es aus diesen Gründen einigen dieser im Kindes- und Jugendalter Sportaktiven schwer, sich auf eine eher freizeit- und breitensportliche Sportaktivität im jungen Erwachsenenalter umzuorientieren und damit die Sportaktivität aufrechtzuerhalten.

Die Differenzierung nach dem Bildungsniveau liefert ebenfalls interessante Effekte. In der 1. Lebensdekade unterscheidet sich die Sportbeteiligung der Gruppe mit einer beruflich-betrieblichen oder schulischen Ausbildung (Lehre, Berufs- oder Handelsschule) kaum von der Gruppe mit einem Fachhochschul- bzw. Hochschulabschluss, Allerdings sind diejenigen mit Fachhochschul- bzw. Hochschulabschluss über alle folgenden Lebensdekaden sportaktiver. Diejenigen ohne beruflichen Abschluss weisen in allen Lebensdekaden das geringste Sportengagement auf. 
Tab. 3 Die 6 Sportkarrieretypen

\begin{tabular}{|c|c|c|c|c|c|c|c|c|}
\hline \multirow[t]{2}{*}{$\begin{array}{l}\text { Sportkarriere- } \\
\text { typen }\end{array}$} & \multirow[t]{2}{*}{$\mathrm{n}$} & \multirow{2}{*}{\multicolumn{2}{|c|}{$\%$}} & $\begin{array}{l}\text { Alter bei } \\
\text { Sport- } \\
\text { einstieg }\end{array}$ & $\begin{array}{l}\text { Sport- } \\
\text { jahre }\end{array}$ & $\begin{array}{l}\text { Anzahl verschie- } \\
\text { dener Haupt- } \\
\text { sportarten }\end{array}$ & $\begin{array}{l}\text { Haupt- } \\
\text { sportart } \\
\text { im Verein }\end{array}$ & $\begin{array}{l}\text { Wettkampf- } \\
\text { orientie- } \\
\text { rung }\end{array}$ \\
\hline & & & & & & \multicolumn{3}{|c|}{ (pro Lebensdekade) } \\
\hline \multirow[t]{2}{*}{ Sportlich Inaktive } & \multirow[t]{2}{*}{453} & \multirow[t]{2}{*}{26,0} & M & 0,00 & 0,00 & 0,00 & 0,00 & 0,00 \\
\hline & & & SD & 0,00 & 0,00 & 0,00 & 0,00 & 0,00 \\
\hline \multirow{2}{*}{$\begin{array}{l}\text { Sportaktive im Kin- } \\
\text { des-/Jugendalter }\end{array}$} & \multirow{2}{*}{296} & \multirow[t]{2}{*}{17,0} & M & 10,51 & 7,22 & 1,00 & 0,89 & 0,53 \\
\hline & & & SD & 3,34 & 3,30 & 0,00 & 0,73 & 0,62 \\
\hline \multirow[t]{2}{*}{ Späteinsteiger } & \multirow[t]{2}{*}{163} & \multirow[t]{2}{*}{9,4} & $M$ & 23,5 & 8,47 & 1,00 & 0,29 & 0,09 \\
\hline & & & SD & 7,02 & 4,98 & 0,00 & 0,58 & 0,28 \\
\hline \multirow{2}{*}{$\begin{array}{l}\text { Sportartentreue } \\
\text { Vereinssportler }\end{array}$} & \multirow[t]{2}{*}{384} & \multirow[t]{2}{*}{22,1} & M & 10,76 & 20,55 & 1,50 & 2,73 & 2,03 \\
\hline & & & SD & 4,36 & 7,93 & 0,50 & 0,77 & 1,08 \\
\hline \multirow{2}{*}{$\begin{array}{l}\text { Vielseitige Vereins- } \\
\text { sportler }\end{array}$} & \multirow{2}{*}{97} & \multirow[t]{2}{*}{5,6} & M & 7,91 & 24,71 & 3,06 & 1,99 & 1,56 \\
\hline & & & SD & 2,56 & 7,2 & 0,28 & 1,17 & 1,27 \\
\hline \multirow{2}{*}{$\begin{array}{l}\text { Nicht-Vereins- } \\
\text { sportler }\end{array}$} & \multirow[t]{2}{*}{346} & \multirow[t]{2}{*}{19,9} & $M$ & 10,64 & 21,77 & 1,64 & 0,47 & 0,41 \\
\hline & & & SD & 4,49 & 8,47 & 0,48 & 0,63 & 0,70 \\
\hline \multirow[t]{2}{*}{ Gesamt } & \multirow[t]{2}{*}{1739} & \multirow[t]{2}{*}{100} & M & 12,08 & 12,27 & 1,09 & 0,99 & 0,72 \\
\hline & & & SD & 6,32 & 11,04 & 0,86 & 1,22 & 1,06 \\
\hline
\end{tabular}

\section{Sportkarrieren in der 1. Lebenshälfte und ihr Einfluss auf das weitere Sportengagement}

Vor dem Hintergrund der individuell unterschiedlichen Sporterfahrungen in der 1. Lebenshälfte wurde eine geeignete Typologisierung dieser Sportkarrieren erarbeitet, um in einem zweiten Schritt deren Einfluss auf das aktuelle Sportengagement differenziert zu überprüfen.

\section{Typologisierung}

Aufgrund der verschiedenen Merkmale, die eine Sportkarriere (in der 1. Lebenshälfte) kennzeichnen, sind zur Typologisierung sowohl ereignisbezogene Merkmale des Sportengagements zu berücksichtigen als auch die Verläufe des Sportengagements abzubilden. Aus diesen Gründen wurde die Clusteranalyse (Ward-Methode) als multivariates Verfahren zur Ermittlung der Sportkarrieren der 1. Lebenshälfte gewählt. In die Clusteranalyse gingen folgende $\mathrm{z}$-standardisierte Variablen ein, die vor dem Hintergrund der theoretischen Überlegungen den Beginn und die Dauer des Sportengagements, die Vereinszugehörigkeit, die Wettkampforientierung sowie die Vielseitigkeit der Sportaktivitäten in der 1. Lebenshälfte beschreiben: (1) das Lebensalter beim Beginn der Sportkarriere, (2) die Anzahl der Sportjahre vom 5.-39. Lebensjahr, (3) die Anzahl der verschiedenen Hauptsportar- ten von der Kindheit bis zur 4. Lebensdekade sowie (4) jeweils die Anzahl der Lebensdekaden, in denen die Hauptsportart im Verein und (5) wettkampforientiert betrieben wurde. Diese Variablen weisen nur geringe Korrelationen untereinander auf $^{4}$. Es wurden insgesamt 6 Sportkarrieretypen für die 1. Lebenshälfte identifiziert (• Tab. 3).

1. Die sportlich Inaktiven sind in der 1. Lebenshälfte im Alter zwischen 5 und 39 Jahren nicht sportaktiv.

2. Die Sportaktiven im Kindes-/Jugendalter haben im Kindesalter ihr Sportengagement begonnen, waren aber nur durchschnittlich 7 Jahre sportaktiv und haben dieses vor oder in der 3. Lebensdekade bereits wieder beendet.

3. Die Späteinsteiger haben erst im jungen Erwachsenenalter (3. Lebensdekade) den Zugang zum Sport gefunden und weisen ein relativ kurzzeitiges Sportengagement von durchschnittlich 8 bis 9 Jahren auf, das kaum durch Wettkampforientierung und nur teilweise durch Vereinsmitgliedschaft gekennzeichnet ist.

\footnotetext{
${ }^{4}$ Lediglich zwischen der Anzahl der Lebensdekaden, in denen die Hauptsportart im Verein, und der Anzahl der Lebensdekaden, in denen die Hauptsportart wettkampforientiert betrieben wurde, besteht ein mittlerer Zusammenhang $(r=0,693)$.
}

4. Die sportartentreuen Vereinssportler betreiben vom Kindes- und Jugendalter an ihre Hauptsportart wettkampforientiert im Verein. Sie sind durchschnittlich in der 1. Lebenshälfte etwa 21 Jahre lang sportaktiv.

5. Die vielseitigen Vereinssportler sind seit dem Kindesalter durchschnittlich 25 Jahre lang im Verein sportlich aktiv. In diesem Zeitraum wechseln sie relativ häufig (durchschnittlich zweimal) die Hauptsportart, die sie teilweise wettkampforientiert ausüben.

6. Die Nicht-Vereinssportler weisen mit ca. 22 Jahren ebenfalls ein langes Sportengagement auf, das sie im Kindes- und Jugendalter begonnen haben. Die Hauptsportart, die sie selten im Verein und wettkampforientiert ausüben, wechseln sie weniger häufig.

Der Anteil der in der 1. Lebenshälfte sportlich Inaktiven ist bei den weiblichen Befragten mit ca. $31 \%$ höher als bei den männlichen (ca. 19\%). Dagegen ist der Anteil der sportartentreuen Vereinssportler bei den männlichen Befragten (ca. 33\%) mehr als doppelt so groß wie bei weiblichen Befragten (ca. 15\%). Insbesondere die empirischen Werte der Sportinaktiven unter den Frauen (standardisiertes Residuum: 2,9) und der sportartentreuen Vereinssportler unter den Männern (standardisiertes Residuum: 6,2) sind höher als statistisch erwartet. Insgesamt ist der Zusammenhang zwischen Sportkarrieretyp und Geschlecht allerdings als gering zu bewerten. $\mathbf{T}$ Tab. 4 verdeutlicht, dass alle Sportkarrieretypen in allen Alterskohorten vertreten sind. Der Anteil der in der 1. Lebenshälfte sportlich Inaktiven ist bei der jüngsten Alterskohorte der 50- bis 59-Jährigen am geringsten und der Anteil an Vereinssportlern am höchsten. Die Anzahl der Sportinaktiven liegt in der jüngsten Alterskohorte unter dem statistisch erwarteten Wert (standardisiertes Residuum $=-4,2$ ) und in der ältesten Alterskohorte darüber (standardisiertes Residuum: 3,9). Zudem liegt die Sportbeteiligungsquote der 50- bis 59 -jährigen sportartentreuen Vereinssportler über dem statistisch erwarteten Wert (standardisiertes Residuum: 2,7). Im Gegensatz zu den älteren Alterskohorten haben die 50- bis 59-Jährigen ihre 1. Lebenshälf- 


\begin{tabular}{|c|c|c|c|c|c|c|c|c|c|c|c|}
\hline \multirow{2}{*}{$\begin{array}{l}\text { Sportkarriere- } \\
\text { typen }\end{array}$} & & \multicolumn{2}{|c|}{ Geschlecht } & \multicolumn{3}{|c|}{ Alterskohorten } & \multicolumn{2}{|c|}{ Wohnort } & \multicolumn{3}{|c|}{ Bildungsabschluss $^{\mathrm{a}}$} \\
\hline & & Weiblich & Männlich & $\begin{array}{l}50-\text { bis } \\
59-J a ̈ h- \\
\text { rige }\end{array}$ & $\begin{array}{l}60-\text { bis } \\
69-J a ̈ h- \\
\text { rige }\end{array}$ & $\begin{array}{l}70-\text { bis } \\
79-J a ̈ h- \\
\text { rige }\end{array}$ & $\begin{array}{l}\text { Chem- } \\
\text { nitz }\end{array}$ & $\begin{array}{l}\text { Braun- } \\
\text { schweig }\end{array}$ & $\begin{array}{l}\text { Kein Bil- } \\
\text { dungsab- } \\
\text { schluss }\end{array}$ & $\begin{array}{l}\text { Lehre/ } \\
\text { Berufs- } \\
\text { schule }\end{array}$ & $\begin{array}{l}\text { Fachhochschul-/ } \\
\text { Hochschulab- } \\
\text { schluss }\end{array}$ \\
\hline \multirow[t]{2}{*}{ Sportlich Inaktive } & $\mathrm{n}$ & 325 & 128 & 100 & 170 & 183 & 234 & 219 & 56 & 261 & 133 \\
\hline & $\%$ & 30,6 & 18,9 & 17,2 & 26,9 & 34,8 & 26,6 & 25,5 & 50,0 & 27,2 & 20,4 \\
\hline \multirow{2}{*}{$\begin{array}{l}\text { Sportaktive im } \\
\text { Kindes-/Jugend- } \\
\text { alter }\end{array}$} & $\mathrm{n}$ & 195 & 101 & 113 & 98 & 85 & 180 & 116 & 20 & 174 & 101 \\
\hline & $\%$ & 18,3 & 14,9 & 19,4 & 15,5 & 16,2 & 20,4 & 13,5 & 17,9 & 18,1 & 15,5 \\
\hline \multirow[t]{2}{*}{ Späteinsteiger } & $\mathrm{n}$ & 109 & 54 & 47 & 64 & 52 & 68 & 95 & 10 & 94 & 57 \\
\hline & $\%$ & 10,3 & 8,0 & 8,1 & 10,1 & 9,9 & 7,7 & 11,1 & 8,9 & 9,8 & 8,7 \\
\hline \multirow{2}{*}{$\begin{array}{l}\text { Sportartentreue } \\
\text { Vereinssportler }\end{array}$} & $\mathrm{n}$ & 159 & 225 & 159 & 137 & 88 & 176 & 208 & 7 & 217 & 155 \\
\hline & $\%$ & 15,0 & 33,3 & 27,3 & 21,7 & 16,7 & 20,0 & 24,2 & 6,3 & 22,6 & 23,7 \\
\hline \multirow{2}{*}{\begin{tabular}{|l} 
Vielseitige \\
Vereinssportler
\end{tabular}} & $\mathrm{n}$ & 49 & 48 & 42 & 28 & 27 & 35 & 62 & 1 & 45 & 51 \\
\hline & $\%$ & 4,6 & 7,1 & 7,2 & 4,4 & 5,1 & 4,0 & 7,2 & 0,9 & 4,7 & 7,8 \\
\hline \multirow{2}{*}{$\begin{array}{l}\text { Nicht-Vereins- } \\
\text { sportler }\end{array}$} & $\mathrm{n}$ & 226 & 120 & 121 & 134 & 91 & 188 & 158 & 18 & 169 & 156 \\
\hline & $\%$ & 21,3 & 17,8 & 20,8 & 21,2 & 17,3 & 21,3 & 18,4 & 16,1 & 17,6 & 23,9 \\
\hline \multirow[t]{3}{*}{ Gesamt } & $\mathrm{n}$ & 1063 & 676 & 582 & 631 & 526 & 881 & 858 & 112 & 960 & 653 \\
\hline & $\%$ & 100,0 & 100,0 & 100,0 & 100,0 & 100,0 & 100,0 & 100,0 & 100,0 & 100,0 & 100,0 \\
\hline & & \multicolumn{2}{|c|}{$\begin{array}{l}\mathrm{Chi}^{2}(5, N=1739)=96,568, \\
\mathrm{p}<0,0005, \\
C l=0,236\end{array}$} & \multicolumn{3}{|c|}{$\begin{array}{l}\mathrm{Chi}^{2}(10, N=1739)=58,462 \\
\mathrm{p}<0,0005 \\
C l=0,130\end{array}$} & \multicolumn{2}{|c|}{$\begin{array}{l}\mathrm{Chi}^{2}(5, N=1739)=31,291 \\
\mathrm{p}<0,0005, \\
C l=0,134\end{array}$} & \multicolumn{3}{|c|}{$\begin{array}{l}\operatorname{Chi}^{2}(15, N=1739)=72,478, \\
\mathrm{p}<0,0005 \\
C l=0,118\end{array}$} \\
\hline
\end{tabular}

te in der zweiten Hälfte des 20. Jahrhunderts verbracht, in welcher der Vereinssport zunehmend an Bedeutung gewann. Die unterschiedliche Entwicklung der beiden Sportsysteme in Ost- und Westdeutschland wirkt sich in der 1. Lebenshälfte der Befragten kaum auf die individuellen Sportkarrieren aus (• Tab. 4). Lediglich der Anteil der Sportaktiven im Kindes-/Jugendalter ist in Chemnitz gröBer als in Braunschweig (standardisiertes Residuum: 2,5). In Chemnitz gelang es unter dem ostdeutschen Sportsystem wenigen Sportaktiven, ihr Sportengagement von der Kindheit und Jugendphase ins Erwachsenenalter aufrechtzuerhalten. Mit einer höheren beruflichen Qualifikation sinkt der Anteil der Sportinaktiven. In der Gruppe ohne beruflichen Abschluss ist die Anzahl der sportlich Inakti$v e n$ in der 1. Lebenshälfte höher (standardisiertes Residuum: 5,0 ) und in der Gruppe derjenigen mit Fachhochschul- bzw. Hochschulabschluss geringer (standardisiertes Residuum: $-2,8$ ) als statistisch erwartet. Die empirischen Werte der NichtVereinssportler mit Fachhochschul- bzw. Hochschulabschluss (standardisiertes Residuum: 2,3) sowie der vielseitigen Vereinssportler mit Fachhochschul- bzw. Hoch- schulabschluss (standardisiertes Residuum: 2,4 ) sind dagegen höher als statistisch erwartet ( Tab. 4).

\section{Einfluss der Sportkarrieretypen auf die aktuelle Sportaktivität}

Zur Analyse der Fragestellung, inwieweit das aktuelle Sportengagement im mittleren und späteren Erwachsenenalter von der Sportkarriere in der 1. Lebenshälfte beeinflusst wird, wurde zunächst der Zusammenhang zwischen den Sportkarrieretypen und der aktuellen Sportaktivität geprüft (• Tab. 5). Hierbei zeigt sich ein signifikanter, aber geringer Zusammenhang $\left(\chi^{2}(5 ; 1739)=75,470 ; p<0,0005\right.$; $\mathrm{CI}=0,208)$. Nur jeder zweite der in der 1. Lebenshälfte sportlich Inaktiven und der Sportaktiven im Kindes-/Jugendalter ist aktuell sportlich aktiv. Demgegenüber treiben fast drei Viertel der Nicht-Vereinssportler, der sportartentreuen Vereinssportler und der vielseitigen Vereinssportler aktuell Sport ( Tab. 5). Damit bestätigt sich die Vermutung der verlaufsbezogenen Abhängigkeit des Sportengagements über die Lebensspanne hinweg. Allerdings hat durch die gewachsene Bedeutung von Bewegung und Sport auch die Hälfte der in der 1. Lebenshälfte sportlich
Inaktiven den Zugang zum Sport in der 2. Lebenshälfte gefunden. Differenziert man die Beteiligungsquoten der Sportkarrieretypen nach Geschlecht, so zeigt sich, dass bei den Frauen bei allen Sportkarrieretypen mit Ausnahme der Sportinaktiven der Anteil der aktuell Sportaktiven größer ist als bei den befragten Männern. Insgesamt unterscheidet sich die Anzahl an Sportaktiven zwischen den Geschlechtern und den Sportkarrieretypen signifikant voneinander (『 Tab. 5). Da insgesamt die sportartentreuen Vereinssportler unter den Männern häufiger vertreten sind als bei den Frauen ( $\mathbf{0}$ Tab. 5), trägt insbesondere die Anzahl an derzeit sportaktiven, männlichen sportartentreuen Vereinssportlern zum signifikanten Geschlechtsunterschied zwischen den derzeit sportaktiven Sportkarrieretypen bei (standardisiertes Residuum: 4,3). Der prozentuale Anteil an derzeit Sportaktive ist jedoch unter den weiblichen sportartentreuen Vereinssportlerinnen höher als bei den männlichen. Dieser Befund ist vermutlich dadurch zu begründen, dass die weiblichen sportartentreuen Vereinssportlerinnen eher sog. Lifetime-Sportarten wie z. B. Schwimmen oder Gymnastik als Hauptsportarten der 1. Lebenshälfte an- 


\section{Hauptbeiträge}

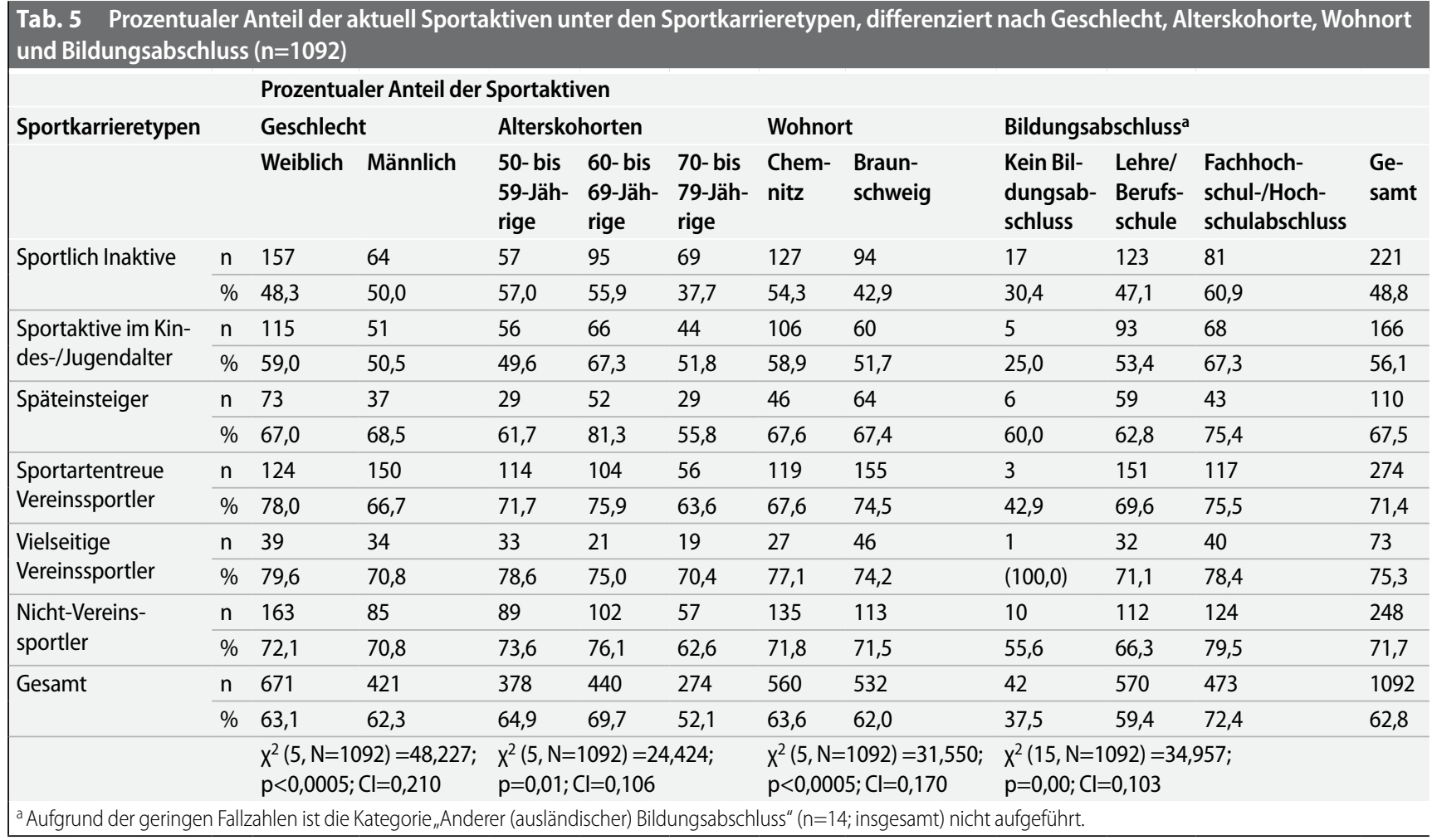

Tab.6 Quotient aus Sportjahren und Lebensjahren ab dem 40. Lebensjahr, differenziert nach Sportkarrieretypen $(n=1739)$

\begin{tabular}{llr} 
& M & SD \\
Sportlich Inaktive & 0,20 & 0,28 \\
$\begin{array}{l}\text { Sportaktive im Kin- } \\
\text { des-/Jugendalter }\end{array}$ & 0,29 & 0,32 \\
\hline Späteinsteiger & 0,50 & 0,40 \\
\hline $\begin{array}{l}\text { Sportartentreue } \\
\text { Vereinssportler }\end{array}$ & 0,58 & 0,40 \\
\hline $\begin{array}{l}\text { Vielseitige } \\
\text { Vereinssportler }\end{array}$ & 0,73 & 0,34 \\
\hline Nicht-Vereinssportler & 0,64 & 0,38 \\
\hline Gesamt & 0,44 & 0,40 \\
\hline Varianzanalyse & $\begin{array}{l}\mathrm{F}(5,1739)=102,09 ; \\
\end{array}$ & $\mathrm{p}<0,0005 ; \eta^{2}=0,23$
\end{tabular}

Lesebeispiel: Die sportartentreuen Vereinssportler sind im Durchschnitt 58\% ihrer Lebensjahre $(M=0,58)$ ab dem 40 . Lebensjahr sportaktiv das entspricht etwa $11 \pm 7,6$ sportaktive Jahre für einen 58-jährigen sportartentreuen Vereinssportler.

gaben, während die männlichen Befragten eher Mannschaftssportarten nannten, die nur sehr selten bis ins späte Erwachsenenalter betrieben werden können (z. B. Fußball). Den männlichen sportartentreuen Vereinssportlern, die am häufigs- ten Fußball als Hauptsportart angaben, ist es aus diesen Gründen vermutlich weniger erfolgreich gelungen, ihre Sportaktivität bis zum Befragungszeitpunkt aufrechtzuerhalten. Der hierzu erforderliche Hauptsportartenwechsel bereitete ihnen wohl Schwierigkeiten (• Tab. 5). Während in den beiden jüngeren Alterskohorten etwa zwei Drittel derzeit regelmäßig eine sportliche Aktivität ausüben, ist nur jeder zweite der ältesten Alterskohorte aktuell sportaktiv. Die Anzahl der 50- bis 59-jährigen Sportinaktiven, die in der 2. Lebenshälfte den Zugang zum Sport gefunden haben und derzeit sportaktiv sind, liegt über dem erwarteten statistischen Wert (standardisiertes Residuum: -2,2). Bei der ältesten Alterskohorte ist der Anteil der aktuell Sportaktiven unter den in der 1. Lebenshälfte sportlich Inaktiven im Vergleich zu den jüngeren deutlich geringer. Unter den $70-$ bis 79-Jährigen gibt es also im Vergleich zu den jüngeren Alterskohorten weniger Wieder- und Neueinsteiger, die erst im Erwachsenenalter den Zugang zum Sport gefunden haben. Die beiden jüngeren Alterskohorten unterscheiden sich v. a. in Bezug auf die aktuelle Sportbeteiligung unter den Späteinsteigern: mit rund $81 \%$ liegt sie bei den 60 - bis 69 -Jährigen höher als bei der jüngsten Alterskohorte (- Tab. 5). Im Vergleich zu Braunschweig sind die sportartentreuen Vereinssportler aus Chemnitz aktuell weniger häufig sportaktiv. Im Gegensatz dazu treiben die bis zum 39. Lebensjahr sportlich Inaktiven aus Chemnitz aktuell häufiger Sport als in Braunschweig. Die Anzahl an Sportaktiven im Kindes-/Jugendalter aus Chemnitz, die wieder eingestiegen sind und derzeit sportaktiv sind, liegt über dem statistisch zu erwartenden Wert (standardisiertes Residuum: 2,3).

Differenziert man die Stichprobe anhand des Bildungsniveaus, so wird wiederum der Zusammenhang zwischen höherer Bildung und Sportengagement bestätigt. Über alle Sportkarrieretypen hinweg weisen diejenigen mit Fachhochschulbzw. Hochschulabschluss die höchste Sportbeteiligung auf. Allerdings liegt die aktuelle Sportbeteiligung der Sportinaktiven ohne Bildungsabschluss höher als der statistisch zu erwartende Wert (standardisiertes Residuum=2.9). 0 Tab. 5 verdeutlicht, dass sich die Sportkarrieretypen in Bezug auf die aktuelle Sportaktivität signifikant unterscheiden und damit in allen Untergruppen lebenszeitliche Abhän- 


\begin{tabular}{|c|c|c|c|c|c|c|c|c|c|}
\hline \multirow{3}{*}{\multicolumn{2}{|c|}{ Sportkarrieretypen }} & \multicolumn{8}{|c|}{ Relative Sportjahre } \\
\hline & & \multicolumn{2}{|c|}{ Geschlecht } & \multicolumn{2}{|l|}{ Wohnort } & \multicolumn{4}{|c|}{ Bildungsabschluss $^{\mathrm{a}}$} \\
\hline & & $\begin{array}{l}\text { Weib- } \\
\text { lich } \\
(n=360)\end{array}$ & $\begin{array}{l}\text { Männ- } \\
\text { lich } \\
(n=222)\end{array}$ & $\begin{array}{l}\text { Chem- } \\
\text { nitz } \\
(n=286)\end{array}$ & $\begin{array}{l}\text { Braun- } \\
\text { schweig } \\
(n=296)\end{array}$ & $\begin{array}{l}\text { Kein Bildungs- } \\
\text { abschluss } \\
(n=14)\end{array}$ & $\begin{array}{l}\text { Lehre/Berufs- } \\
\text { schule } \\
(n=328)\end{array}$ & $\begin{array}{l}\text { Fach-/Hoch- } \\
\text { schulabschluss } \\
(n=235)\end{array}$ & $\begin{array}{l}\text { Gesamt } \\
(n=582)\end{array}$ \\
\hline \multirow[t]{2}{*}{ Sportlich Inaktive } & M & 0,282 & 0,215 & 0,284 & 0,242 & $0,291^{*}$ & 0,231 & 0,343 & 0,266 \\
\hline & SD & 0,321 & 0,313 & 0,305 & 0,338 & 0,274 & 0,294 & 0,364 & 0,319 \\
\hline \multirow{2}{*}{$\begin{array}{l}\text { Sportaktive im Kin- } \\
\text { des-/Jugendalter }\end{array}$} & M & 0,319 & 0,178 & 0,278 & 0,262 & $0,000^{*}$ & 0,228 & 0,390 & 0,271 \\
\hline & SD & 0,346 & 0,292 & 0,335 & 0,337 & 0,000 & 0,306 & 0,376 & 0,334 \\
\hline \multirow[t]{2}{*}{ Späteinsteiger } & M & 0,452 & 0,409 & 0,260 & 0,483 & $0,500^{*}$ & 0,412 & 0,462 & 0,436 \\
\hline & SD & 0,425 & 0,400 & 0,381 & 0,412 & 0,707 & 0,390 & 0,438 & 0,412 \\
\hline \multirow{2}{*}{$\begin{array}{l}\text { Sportartentreue } \\
\text { Vereinssportler }\end{array}$} & M & 0,552 & 0,578 & 0,518 & 0,613 & $0,519^{*}$ & 0,549 & 0,594 & 0,567 \\
\hline & SD & 0,420 & 0,410 & 0,423 & 0,400 & 0,501 & 0,403 & 0,427 & 0,413 \\
\hline \multirow{2}{*}{$\begin{array}{l}\text { Vielseitige } \\
\text { Vereinssportler }\end{array}$} & M & 0,772 & 0,642 & 0,602 & 0,784 & $-b$ & 0,792 & 0,683 & 0,719 \\
\hline & SD & 0,323 & 0,394 & 0,439 & 0,290 & - & 0,310 & 0,378 & 0,356 \\
\hline \multirow[t]{2}{*}{ Nicht-Vereinssportler } & M & 0,648 & 0,535 & 0,623 & 0,612 & $0,000^{c}$ & 0,547 & 0,715 & 0,618 \\
\hline & SD & 0,399 & 0,426 & 0,392 & 0,426 & - & 0,419 & 0,370 & 0,407 \\
\hline \multirow[t]{2}{*}{ Gesamt } & M & 0,478 & 0,454 & 0,436 & 0,500 & 0,266 & 0,418 & 0,555 & 0,469 \\
\hline & SD & 0,408 & 0,416 & 0,401 & 0,418 & 0,381 & 0,399 & 0,413 & 0,410 \\
\hline \multicolumn{10}{|l|}{ Varianzanalyse } \\
\hline Sportkarrieretyp & \multicolumn{5}{|c|}{$F(5,582)=6,18 ; p<0,0005 ; \eta^{2}=0,06$} & \multicolumn{2}{|c|}{ Geschlecht $\times$ Bildungsabschluss } & \multicolumn{2}{|c|}{$F(3,582)=1,28 ; p=0,28 ; \eta^{2}=0,01$} \\
\hline Geschlecht & \multicolumn{5}{|c|}{$F(1,582)=1,97 ; p=0,16 ; \eta^{2}=0,00$} & \multicolumn{2}{|c|}{ Wohnort $\times$ Bildungsabschluss } & \multicolumn{2}{|c|}{$F(2,582)=1,19 ; p=0,31 ; \eta^{2}=0,01$} \\
\hline Wohnort & \multicolumn{5}{|c|}{$F(1,582)=1,74 ; p=0,18 ; \eta^{2}=0,00$} & \multicolumn{2}{|c|}{ Sportkarrieretyp $\times$ Geschlecht $\times$ Wohnort } & \multicolumn{2}{|c|}{$F(5,582)=0,68 ; p=0,64 ; \eta^{2}=0,01$} \\
\hline Bildungsabschluss & \multicolumn{5}{|c|}{$F(3,582)=3,15 ; p=0,03 ; n^{2}=0,02$} & \multicolumn{2}{|c|}{$\begin{array}{l}\text { Sportkarrieretyp } \times \text { Geschlecht } \times \text { Bildungs- } \\
\text { abschluss }\end{array}$} & \multicolumn{2}{|c|}{$F(6,582)=0,59 ; p=0,74 ; \eta^{2}=0,01$} \\
\hline $\begin{array}{l}\text { Sportkarrieretyp } \times \\
\text { Geschlecht }\end{array}$ & \multicolumn{4}{|c|}{$F(5,582)=0,76 ; p=0,58 ; \eta^{2}=0,01$} & & \multicolumn{2}{|c|}{$\begin{array}{l}\text { Sportkarrieretyp } \times \text { Wohnort } \times \text { Bildungsab- } \\
\text { schluss }\end{array}$} & \multicolumn{2}{|c|}{$F(6,582)=0,85 ; p=0,53 ; \eta^{2}=0,01$} \\
\hline $\begin{array}{l}\text { Sportkarriere- } \\
\text { typ } \times \text { Wohnort }\end{array}$ & \multicolumn{4}{|c|}{$F(5,582)=1,22 ; p=0,30 ; \eta^{2}=0,01$} & & \multicolumn{2}{|c|}{ Geschlecht $\times$ Wohnort $\times$ Bildungsabschluss } & \multicolumn{2}{|c|}{$F(1,582)=0,02 ; p=0,89 ; \eta^{2}=0,00$} \\
\hline $\begin{array}{l}\text { Sportkarrieretyp } \times \\
\text { Bildungsabschluss }\end{array}$ & $\mathrm{F}($ & $(11,582)=1$ & $07 ; p=0,38$ & $; \eta^{2}=0,02$ & & $\begin{array}{l}\text { Sportkarrierety } \\
\text { ort } \times \text { Bildungsa }\end{array}$ & lecht $\times$ Wohn- & $F(5,582)=1,84 ; p=0$, & $0 ; \eta^{2}=0,0$ \\
\hline Geschlecht $\times$ Wohnort & F( & $(1,582)=1,4$ & $7 ; p=0,23$ & $\eta^{2}=0,00$ & & & & & \\
\hline $\begin{array}{l}\text { a Aufgrund der geringen } F \\
{ }^{b} n=0 ;{ }^{c} n=0 ;{ }^{*} n \leq 10 .\end{array}$ & Fallzah & hlen ist die $K_{c}$ & ategorie „A $\mathrm{Al}_{\mathrm{l}}$ & erer (auslä & cher) Bi & gsabschluss" (n & eführt. & & \\
\hline
\end{tabular}

gigkeiten des Sportengagements erkennbar sind.

\section{Einfluss der Sportkarrieretypen auf den Verlauf des Sportengagements}

Die Sportkarrieretypen unterscheiden sich vermutlich nicht nur in Bezug auf den Einfluss auf die aktuelle Sportaktivität, sondern auch in Bezug auf die Beeinflussung des Verlaufs des Sportengagements in der 2. Lebenshälfte. Dieser wurde anhand der relativen Anzahl an sportaktiven Lebensjahren in der 2. Lebenshälfte ab dem 40. Lebensjahr (• Tab. 6) gemessen. Die varianzanalytische Überprüfung (einfaktorielle ANOVA) der Mittelwertunterschiede ergibt signifikante Unterschiede. Die Effekte sind nach Co- hen (1988, S. 284-288) als groß zu interpretieren. Im paarweisen Post-hoc-Vergleich (Tamhane-Test) unterscheiden sich die sportlich Inaktiven und Sportaktiven im Kindes-/Jugendalter signifikant von den anderen Sportkarrieretypen.

Die vielseitigen Vereinssportler weisen verlaufsbezogen die größte Sportaktivität auf und sind fast während drei Viertel ihrer Lebenszeit in der 2. Lebenshälfte sportlich aktiv. Das geringste verlaufsbezogene Sportengagement in der 2. Lebenshälfte zeigt sich bei den Sportaktiven im Kindes-/Jugendalter und v. a. bei den sportlich Inaktiven (• Tab. 6).

Aufgrund der unterschiedlich langen Zeitspannen der 2. Lebenshälfte (z. B. 30-39 Jahre für die 70- bis 79-Jährigen) soll im Folgenden die Dauer der Aufrechterhaltung der Sportaktivität differenziert nach Alterskohorten betrachtet werden. Für alle 3 Alterskohorten wurde hierzu eine 4-faktorielle Varianzanalyse (ANOVA) gerechnet. In die Berechnung gingen die Sportkarrieretypen als 6-stufiger Faktor, das Bildungsniveau als 4-stufiger Faktor sowie das Geschlecht und der Wohnort als 2-stufiger Faktor ein. Als abhängige Variable wurde die Anzahl der Sportjahre in Relation zum Lebensalter berücksichtigt.

Die Alterskohorte der 50- bis 59-Jährigen. Die Varianzanalyse für die jüngste Alterskohorte der 50- bis 59-Jährigen ergab einen signifikanten Einfluss 


\section{Hauptbeiträge}

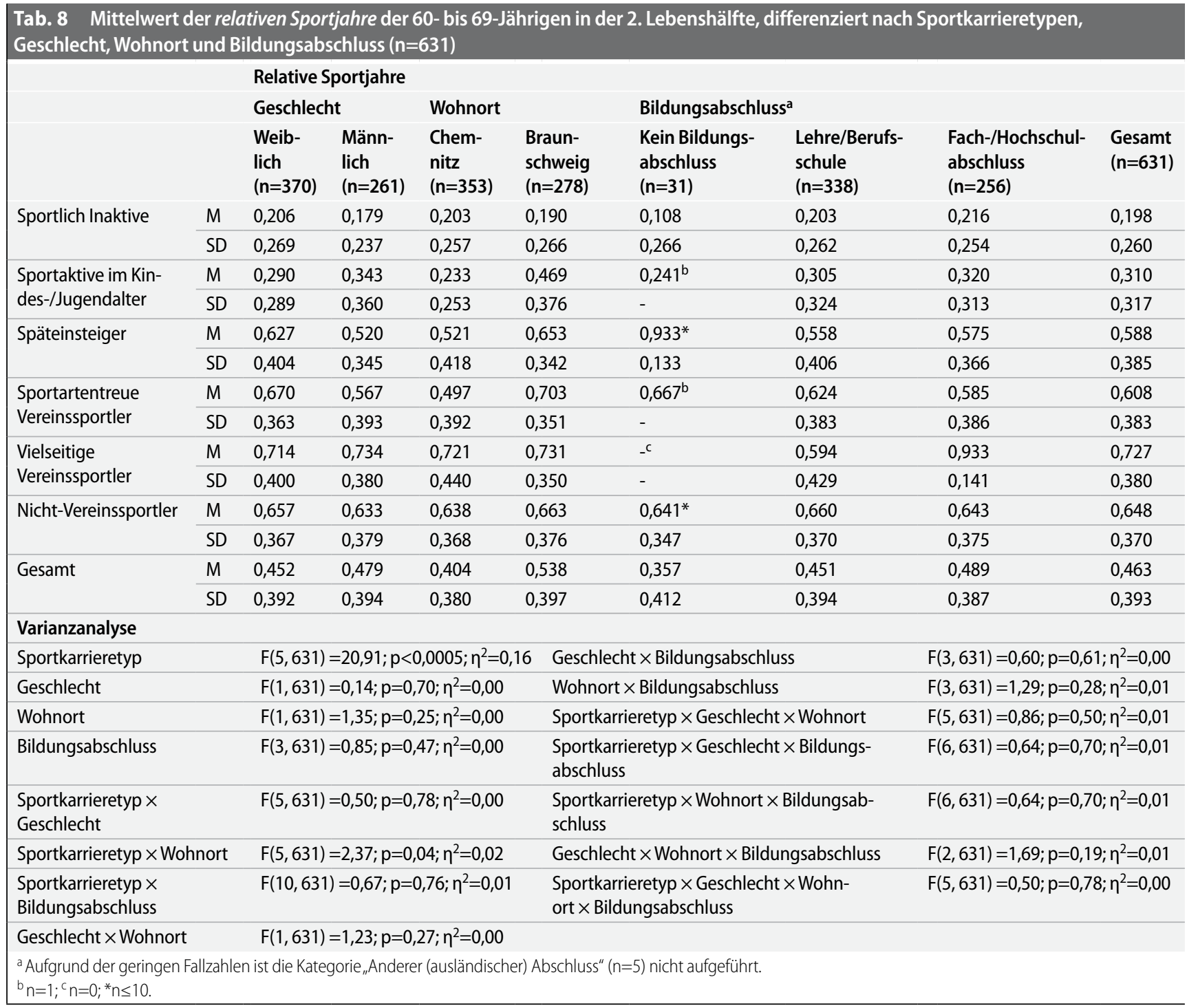

der Sportkarrieretypen auf den Verlauf der Sportaktivität in der 2. Lebenshälfte (• Tab. 7). Lediglich die vielseitigen Vereinssportler weisen signifikante Unterschiede zu den sportlich Inaktiven sowie den kurzzeitig sportaktiven Späteinsteigern und Sportaktiven im Kindes-/Jugendalter auf (Scheffé-Test).

Die geschlechtsspezifischen Unterschiede sind nicht signifikant und die Differenzen zwischen den Sportkarrieretypen sind ähnlich (keine Wechselwirkung zwischen den Faktoren Geschlecht und Sportkarrieretyp). Die Frauen der jüngsten Alterskohorte sind jedoch über alle Sportkarrieretypen hinweg in der 2. Lebenshälfte tendenziell sportaktiver als die Männer (• Tab. 7). Bei der Differenzierung nach dem Wohnort ergeben sich wiederum keine signifikanten Unterschiede. Die vielseitigen Vereinssportler, die sportartentreuen Vereinssportler sowie die Späteinsteiger der Teilstichprobe Braunschweig sind in der 2. Lebenshälfte etwas länger sportaktiv (• Tab. 7). Dagegen sind die Unterschiede zwischen den verschiedenen Bildungsabschlüssen signifikant. Wiederum wird die These bestätigt, dass mit zunehmendem Bildungsniveau die Sportbeteiligung steigt. Über fast alle Sportkarrieretypen weisen diejenigen mit einem Fachhochschul- bzw. Hochschulabschluss ein längeres Sportengagement in der 2. Lebenshälfte auf. Die meisten relativen Sportjahre haben die vielseitigen Vereinssportler mit einer betrieblichen bzw. Berufsausbildung (Lehre. Berufs- bzw. Handelsschule; 0 Tab. 7).
Die Alterskohorte der 60- bis 69-Jährigen. Auch die Varianzanalyse für die Alterskohorte der 60- bis 69-Jährigen ergab, dass der Verlauf der Sportaktivität in der 2. Lebenshälfte signifikant von den Sportkarrieretypen der 1. Lebenshälfte beeinflusst wird ( $\bullet$ Tab. 8). Die sportlich Inaktiven und die Sportaktiven im Kindes-/Jugendalter unterscheiden sich nach dem paarweisen Post-hocVergleich (Scheffé-Test) von allen anderen Sportkarrieretypen. Die geschlechtsspezifischen Unterschiede sind nicht signifikant. Allerdings weisen die weiblichen sportartentreuen Vereinssportlerinnen und die Späteinsteigerinnen eine dauerhaftere Sportaktivität im mittleren Erwachsenenalter auf. Der Faktor Wohnort hat zwar einen signifikanten Einfluss 


\begin{tabular}{|c|c|c|c|c|c|c|c|c|c|c|c|c|}
\hline & & \multicolumn{11}{|c|}{ Relative Sportjahre } \\
\hline & & \multicolumn{3}{|c|}{ Geschlecht } & \multicolumn{3}{|l|}{ Wohnort } & \multicolumn{5}{|l|}{ Bildungsabschluss $^{a}$} \\
\hline & & $\begin{array}{l}\text { Weiblic } \\
(n=333\end{array}$ & & $\begin{array}{l}\text { Männlich } \\
(n=193)\end{array}$ & $\begin{array}{l}\text { Chemnitz } \\
(n=242)\end{array}$ & $\begin{array}{l}\text { Brau } \\
\text { schw } \\
(n=2\end{array}$ & & $\begin{array}{l}\text { Kein Bildungsab- } \\
\text { schluss } \\
(n=67)\end{array}$ & $\begin{array}{l}\text { Lehre/Berufss } \\
(n=294)\end{array}$ & chule & $\begin{array}{l}\text { Fach-/Hoch- } \\
\text { schulabschluss } \\
(n=162)\end{array}$ & $\begin{array}{l}\text { Gesamt } \\
(n=526)\end{array}$ \\
\hline \multirow{2}{*}{$\begin{array}{l}\text { Sportlich } \\
\text { Inaktive }\end{array}$} & $M$ & 0,171 & & 0,150 & 0,114 & 0,200 & & 0,145 & 0,162 & & 0,193 & 0,165 \\
\hline & SD & 0,279 & & 0,236 & 0,206 & 0,298 & & 0,255 & 0,272 & & 0,268 & 0,267 \\
\hline \multirow{2}{*}{$\begin{array}{l}\text { Sportaktive im } \\
\text { Kindes-/Jugend- } \\
\text { alter }\end{array}$} & $M$ & 0,290 & & 0,273 & 0,292 & 0,274 & & 0,162 & 0,270 & & 0,369 & 0,285 \\
\hline & SD & 0,320 & & 0,316 & 0,295 & 0,35 & & 0,315 & 0,325 & & 0,293 & 0,317 \\
\hline \multirow[t]{2}{*}{ Späteinsteiger } & $M$ & 0,438 & & 0,505 & 0,473 & 0,435 & & $0,469^{*}$ & 0,463 & & 0,410 & 0,454 \\
\hline & SD & 0,381 & & 0,423 & 0,406 & 0,378 & & 0,480 & 0,387 & & 0,432 & 0,389 \\
\hline \multirow{2}{*}{$\begin{array}{l}\text { Sportartentreue } \\
\text { Vereinssportler }\end{array}$} & M & 0,517 & & 0,564 & 0,410 & 0,637 & & $0,717^{*}$ & 0,507 & & 0,582 & 0,544 \\
\hline & SD & 0,407 & & 0,394 & 0,376 & 0,38 & & 0,330 & 0,397 & & 0,406 & 0,398 \\
\hline \multirow{2}{*}{$\begin{array}{l}\text { Vielseitige } \\
\text { Vereinssportler }\end{array}$} & $M$ & 0,791 & & 0,719 & 0,859 & 0,705 & & 0,564 & 0,664 & & 0,833 & 0,756 \\
\hline & SD & 0,202 & & 0,372 & 0,188 & 0,326 & & $-b$ & 0,378 & & 0,165 & 0,293 \\
\hline \multirow{2}{*}{$\begin{array}{l}\text { Nicht-Vereins- } \\
\text { sportler }\end{array}$} & $M$ & 0,741 & & 0,554 & 0,583 & 0,744 & & $0,703^{*}$ & 0,658 & & 0,658 & 0,663 \\
\hline & SD & 0,345 & & 0,378 & 0,410 & 0,306 & & 0,351 & 0,378 & & 0,372 & 0,369 \\
\hline \multirow[t]{2}{*}{ Gesamt } & M & 0,378 & & 0,418 & 0,350 & 0,425 & & 0,283 & 0,373 & & 0,474 & 0,393 \\
\hline & SD & 0,390 & & 0,392 & 0,376 & 0,401 & & 0,369 & 0,391 & & 0,390 & 0,391 \\
\hline \multicolumn{13}{|l|}{ Varianzanalyse } \\
\hline \multicolumn{3}{|l|}{ Sportkarrieretyp } & \multicolumn{3}{|c|}{$\begin{array}{l}F(5,526)=13,23 ; p<0,0005 \\
\eta^{2}=0,13\end{array}$} & \multicolumn{4}{|c|}{ Geschlecht $\times$ Bildungsabschluss } & \multicolumn{3}{|c|}{$F(2,526)=2,54 ; p=0,08 ; \eta^{2}=0,01$} \\
\hline \multicolumn{3}{|l|}{ Geschlecht } & \multicolumn{4}{|c|}{$F(1,526)=0,96 ; p=0,33 ; \eta^{2}=0,00$} & \multicolumn{3}{|c|}{ Wohnort $\times$ Bildungsabschluss } & \multicolumn{3}{|c|}{$F(3,526)=0,23 ; p=0,87 ; \eta^{2}=0,00$} \\
\hline \multicolumn{3}{|l|}{ Wohnort } & \multicolumn{4}{|c|}{$F(1,526)=0,25 ; p=0,62 ; \eta^{2}=0,00$} & \multicolumn{3}{|c|}{ Sportkarrieretyp $\times$ Geschlecht $\times$ Wohnort } & \multicolumn{3}{|c|}{$F(5,526)=0,10 ; p=0,99 ; \eta^{2}=0,00$} \\
\hline \multicolumn{3}{|l|}{ Bildungsabschluss } & \multicolumn{4}{|c|}{$F(3,526)=0,82 ; p=0,48 ; \eta^{2}=0,01$} & \multicolumn{3}{|c|}{$\begin{array}{l}\text { Sportkarrieretyp } \times \text { Geschlecht } \times \text { Bildungs- } \\
\text { abschluss }\end{array}$} & \multicolumn{3}{|c|}{$F(8,526)=0,64 ; p=0,74 ; \eta^{2}=0,01$} \\
\hline \multicolumn{3}{|c|}{ Sportkarrieretyp $\times$ Geschlecht } & $\mathrm{F}(5$, & $526)=2,58 ; p$ & $=0,03 ; \eta^{2}=0$, & & & $\begin{array}{l}\text { karrieretyp } \times \text { Wohnor } \\
\text { luss }\end{array}$ & $\times$ Bildungs- & $F(8,5$ & 26) $=2,18 ; p=0,03 ; n$ & $\eta^{2}=0,04$ \\
\hline Sportkarrieretyp > & Woh & & $\mathrm{F}(5$, & 526) $=2,54 ; \mathrm{p}$ & $=0,03 ; \eta^{2}=0$, & & Ges & lecht $\times$ Wohnort $\times$ Bil & ungsabschluss & $F(1,5$ & 26) $=0,16 ; p=0,69 ; n$ & $\eta^{2}=0,00$ \\
\hline $\begin{array}{l}\text { Sportkarrieretyp } \\
\text { Bildungsabschlus }\end{array}$ & & & $\mathrm{F}(11$ & $1,526)=0,66$ & $p=0,78 ; \eta^{2}=0$ & & & $\begin{array}{l}\text { karrieretyp } \times \text { Geschle } \\
\text { Bildungsabschluss }\end{array}$ & t $\times$ Wohn- & $F(4,5$ & 26) $=0,74 ; p=0,57 ; n$ & $n^{2}=0,01$ \\
\hline Geschlecht $\times$ Woh & nort & & $\mathrm{F}(1$, & $526)=0,20 ; p$ & $=0,66 ; \eta^{2}=0$, & & & & & & & \\
\hline $\begin{array}{l}\text { Aufgrund der gerin } \\
n=0 ;{ }^{*} n \leq 10 \text {. }\end{array}$ & & ahlen ist & tc & Kategorie, „And & erer (ausländisc & cher) $\mathrm{Bi}$ & & chluss" ( $n=3$ ) nicht au & führt. & & & \\
\hline
\end{tabular}

auf die Sportkarriere der 60- bis 69-Jährigen in der 2. Lebenshälfte, jedoch nur mit einem geringen Effekt. Im Gegensatz zur jüngeren Alterskohorte sind bei den 6o- bis 69-Jährigen in Braunschweig fast alle Sportkarrieretypen in der 2. Lebenshälfte länger sportaktiv. Im Vergleich zu der jüngeren Alterskohorte stand das Sportengagement der 6o- bis 69-Jährigen in der 2. Lebenshälfte eine längere Zeit unter dem Einfluss des ostdeutschen Sportsystems. Durch den Transformationsprozess haben sich jedoch die Sportkarrieren angeglichen, so dass bei der aktuellen Sportaktivität kaum Unterschiede bestehen. Im Gegensatz zur jüngsten Alterskohorte ergeben sich bei den 6o- bis 69-Jährigen hinsichtlich der Bildungsabschlüsse keine signifikanten Unterschiede.

Die Alterskohorte der 70- bis 79-Jährigen. Wie bei den beiden jüngeren Alterskohorten ergibt die Varianzanalyse auch für die älteste Alterskohorte einen signifikanten Einfluss der Sportkarrieretypen auf die verlaufsbezogene Sportaktivität in der 2. Lebenshälfte (• Tab. 9). Beim paarweisen Post-hoc-Test unterscheiden sich die sportlich Inaktiven und Sportaktiven im Kindes-/Jugendalter von den anderen Sportkarrieretypen signifikant. Insbesondere bei den Nicht-Vereinssportler gibt es geschlechtsspezifische Unterschiede. Obwohl in der 1. Lebenshälfte noch geschlechtsspezifische Unter- schiede in der Alterskohorte der 7o- bis 79-Jährigen zu verzeichnen sind, wirken sich diese nicht auf den Verlauf des Sportengagements im mittleren und höheren Erwachsenenalter aus. Bei der Differenzierung nach dem Wohnort besteht wiederum kein signifikanter Interaktionseffekt zwischen den Sportkarrieretypen und dem Wohnort (- Tab. 9). Im Gegensatz zu den jüngeren Alterskohorten haben die in der 1. Lebenshälfte sportlich Inaktiven der Alterskohorte der 70- bis 79-Jährigen aus Chemnitz weniger häufig im mittleren und späteren Erwachsenenalter den Zugang zum Sport gefunden ( $\mathbf{\bullet}$ Tab. 7, 8 und 9) und sie halten ihr Engagement tendenziell weniger lange in der 2. Lebenshälfte aufrecht. Es ist zu vermuten, 
dass im Vergleich zur ältesten Alterskohorte insbesondere die sportlich Inaktiven der jüngeren Alterskohorten aus Chemnitz vom Wechsel des Sportsystems im Sinne eines erleichternden Zugangs zum Sport profitierten. Die unterschiedlichen Ergebnisse bei den anderen Sportkarrieretypen sind hingegen nur schwer zu interpretieren. Wie bei den 60- bis 69-Jährigen ergibt sich bei der ältesten Alterskohorte kein signifikanter Unterschied zwischen den Bildungsgruppen. Es besteht jedoch ein signifikanter Interaktionseffekt zwischen den Sportkarrieretypen, dem Wohnort und den Bildungsabschlüssen. Insgesamt weisen die Sportkarrieretypen in der 2. Lebenshälfte ein längeres Sportengagement auf, die bis zum 40. Lebensjahr viele Jahre sportaktiv waren (vielseitige Vereinssportler, sportartentreue Vereinssportler und Nicht-Vereinssportler), aus Braunschweig stammen und einen Fachhochschul- bzw. Hochschulabschluss erreicht haben.

\section{Ausblick und Fazit}

Ausgehend von den beiden zentralen Fragestellungen ist festzuhalten, dass das Sportengagement in der 2. Lebenshälfte von der sportlichen Vorgeschichte abhängig ist. Sowohl das aktuelle Sportengagement als auch der Verlauf der Sportaktivität im mittleren und späteren Erwachsenenalter werden durch die Sportkarrieretypen der 1. Lebenshälfte beeinflusst. Der Einfluss bezieht sich dabei insbesondere auf die Dauer des Sportengagements in der 2. Lebenshälfte im Sinne der möglichst konstanten Aufrechterhaltung der Sportaktivität. Entgegen der postulierten Annahme ist die Bedeutung eines frühen Einstiegs im Kindes- und Jugendalter eher gering. Er wirkt sich nur dann positiv auf die Sportaktivität in der 2. Lebenshälfte aus, wenn das regelmäßige Sporttreiben im jungen und mittleren Erwachsenenalter aufrechterhalten wird und so zu einem langjährigen Sportengagement führt. Entgegen der vielfach vermuteten Bedeutung des Vereins- und Wettkampfsports für lebenslanges Sporttreiben ist der Einfluss der Vereinsbindung und der Wettkampforientierung auf das Sportengagement in der 2. Lebenshälfte eher als gering zu bewerten. Sowohl die aktuelle
Sportaktivität als auch das verlaufsbezogene Sportengagement der Vereinssportler im mittleren und späteren Erwachsenenalter unterscheiden sich über alle Alterskohorten hinweg nicht signifikant von den Nicht-Vereinssportlern. Eine eher vielseitige Sportkarriere mit einigen Wechseln der Hauptsportarten scheint sich dagegen positiv auf die Sportkarriere in der 2. Lebenshälfte auszuwirken. Über alle Alterskohorten hinweg weisen die vielseitigen Vereinssportler das in Relation zu den Lebensjahren längste Sportengagement im mittleren und späteren Erwachsenenalter auf.

Diese lebenszeitlichen Abhängigkeiten des Sportengagements über die Lebensspanne stehen zudem unter dem moderierenden Einfluss der sozialen Faktoren Geschlecht und Bildungsniveau sowie gesellschaftlicher Rahmenbedingungen. Mit zunehmendem Bildungsniveau steigt die Sportbeteiligung. In den vergangenen 10 Jahren ist die Sportbeteiligung über alle Alterskohorten hinweg gestiegen (Periodeneffekt). Vermutlich aufgrund der sozialen Öffnung und Ausdifferenzierung des Sports hat fast jeder zweite der bis zum 40. Lebensjahr sportlich Inaktiven oder nur kurzeitig Sportaktiven im Kindes-/Jugendalter über alle Alterskohorten hinweg den Neu- bzw. Wiedereinstieg in das Sporttreiben gefunden. Bei den Frauen ist der Anteil an sportlich Inaktiven in der 1. Lebenshälfte zwar größer, jedoch halten diejenigen, die in der 2. Lebenshälfte den Zugang zum Sport finden, das Sportengagement etwas länger aufrecht. Darüber hinaus ist der Einfluss der Kriegs- und Nachkriegsjahre bei den beiden älteren Alterskohorten als Kohorteneffekt zu deuten. Zwar gibt es entgegen der Vermutung nur geringfügige Unterschiede zwischen den Sportkarrieretypen von Chemnitz und Braunschweig, allerdings unterscheiden sie sich bezüglich ihres Einflusses auf die Verläufe des Sportengagements in der 2. Lebenshälfte.

Auf der Grundlage des theoretischmethodischen Konzepts der Lebensverlaufsforschung und sozialisationstheoretischer Überlegungen liefert die vorliegende Untersuchung erste Erkenntnisse über den Verlauf des Sportengagements in der Lebensspanne und Ansätze zur Erklärung des Sportengagements in der 2. Le- benshälfte. Trotz der mit der retrospektiven Datenerhebung verbundenen spezifisch methodischen Probleme (mangelnde Erinnerungsfähigkeit, Tendenz zur biografischen Glättung) entsprechen die in der vorliegenden Studie erhobenen Daten weitestgehend den Befunden, die über kürzere Zeiträume prospektiv erhoben wurden (Breuer, 2003). Um die spezifischen methodischen Schwierigkeiten der retrospektiven Datenerhebung jedoch zu überwinden, wären längerfristig angelegte Längsschnittdesigns anzustreben.

Aufgrund seiner Komplexität konnte der theoretische Bezugsrahmen nicht in seiner Gesamtheit empirisch überprüft werden. In der vorliegenden Studie wurde der Fokus ausschließlich auf die Sportkarriere und die Prüfung ihrer lebenszeitlichen Abhängigkeiten gerichtet. Der Einfluss von gesamtgesellschaftlichen Veränderungen auf die individuellen Sportkarrieren konnte im Rahmen dieses Beitrags lediglich durch die Berücksichtigung der Variablen (Alterskohorte, Geschlecht, Wohnort und Bildungsabschluss) beschrieben werden. Ausgehend von diesen Beobachtungen und Erfahrungen könnte der vorliegende Bezugsrahmen in weiterführenden Arbeiten um theoretische Konzepte zur Analyse der Wechselwirkungen der einzelnen Karrieren in verschiedenen Lebensbereichen und des Einflusses gesamtgesellschaftlicher Veränderungen auf die individuelle Sportkarriere erweitert werden. Auf methodischer Ebene könnte der dargestellte Ansatz um qualitative Einzelfallanalysen ergänzt werden, welche ein vertieftes Verständnis der Zusammenhänge und Wirkmechanismen ermöglichen.

\section{Korrespondenzadresse \\ Claudia Klostermann}

Institut für Sportwissenschaft, Universität Bern Bremgartenstr. 145, 3012 Bern

Schweiz

claudia.engel@ispw.unibe.ch

Interessenkonflikt. Die korrespondierende Autorin gibt an, dass kein Interessenkonflikt besteht.

\section{Literatur}

1. Allmer, H. (1992). Sportliches Handeln im Alter: einsteigen, durchhalten, weitermachen? In H. Baumann (Hrsg.), Altern und körperliches Training (S. 21-38). Bern: Huber. 
2. Allmer, H. (Hrsg.). (2002). Sportengagement im Lebensverlauf. Sankt Augustin: Academia.

3. Backes, G. M. \& Clemens, W. (2008). Lebensphase Alter. Eine Einführung in die sozialwissenschaftliche Altersforschung. Weinheim: Juventa.

4. Baur, J. \& Burrmann, U. (2000). Unerforschtes Land: Jugendsport in ländlichen Regionen. Aachen: Meyer und Meyer.

5. Baur, J. (1989). Körper- und Bewegungskarrieren. Dialektische Analysen zur Entwicklung von Körper und Bewegung im Kindes- und Jugendalter. Schorndorf: Hofmann.

6. Baur, J. (1998). Hochleistungssportliche Karrieren im Nachwuchsbereich. Sportwissenschaft, 28 9-26.

7. Baur, J., Koch, U., Krüger, D., Quilitz, T., Ruge, T. \& Telschow, S. (1996). Seniorensport in Ostdeutschland: zwischen Powersport und Kaffeeklatsch. Aachen: Meyer und Meyer.

8. Becker, S. \& Klein, T. (2007). Altern und Sport - zur Veränderung der sportlichen Aktivität im Lebenslauf. In H.-W. Wahl \& H. Mollenkopf (Hrsg.), Alternsforschung am Beginn des 21. Jahrhunderts (S. 287-305). Berlin: Akademische Verlagsgesellschaft.

9. Becker, S., Klein, T. \& Schneider, S. (2006). Sportaktivität in Deutschland im 10-Jahres-Vergleich:Veränderungen und soziale Unterschiede. Deutsche Zeitschrift für Sportmedizin, 57 (9), 226-232.

10. Beunen, G. P., Lefevre, J., Philippaerts, R. M., Delvaux, K., Thomis, M., Claessens, A. L., Vanreusel, B., Lysens, R., Eynde, B. V. \& Renson, R. (2004). Adolescent Correlates of Physical Activity: A 26-year Follow-up. Medicine \& Science in Sports \& Exercise, 1930-1936.

11. Bortz, J. \& Döring, N. (2006). Forschungsmethoden und Evaluation für Human- und Sozialwissenschaftler (4., überarbeitete Aufl.). Berlin: Springer.

12. Breuer, C., \& Wicker, P. (2007). Körperliche Aktivität über die Lebensspanne. In R. Fuchs, W. Göhner \& H. Seelig (Hrsg.), Aufbau eines körperlich-aktiven Lebensstils. Theorie, Empirie und Praxis (S. 89107). Göttingen: Hogrefe.

13. Breuer, C. \& Wicker, P. (2009). Decreasing sports activity with increasing age? Findings from a 20-year longitudinal and cohort sequence analysis. Research Quarterly for Exercise and Sport, 80 (1), 22-31.

14. Breuer, C. (2003). Entwicklung und Stabilität sportlicher Aktivität im Lebensverlauf. Zur Rolle von Alters-, Perioden- und Kohorteneffekten. Sportwissenschaft, 33 (3), 263-279.

15. Breuer, C. (2004). Zur Dynamik der Sportnachfrage im Lebenslauf. Sport und Gesellschaft, 1, 50-72.

16. Breuer, C. \& Wicker, P. (2008). Demographic and economic factors influencing inclusion in the German sports system. A microanalysis of the years 1985-2005. European Journal for Sport and Society, 5, 35-43.

17. Brinkhoff, K.-P. (1998). Sport und Sozialisation im Jugendalter. Entwicklung, soziale Unterstützung und Gesundheit. Weinheim: Juventa.

18. Brückner, E. (1990). Die retrospektive Erhebung von Lebensverläufen. In K. U. Mayer (Hrsg.), Lebensverläufe und sozialer Wandel (Kölner Zeitschrift für Soziologie und Sozialpsychologie, Sonderheft 31/1990, S. 374-403). Opladen:Westdeutscher Verlag.

19. Cohen, J. (1988). Statistical Power Analysis for the Behavioral Sciences (2nd ed.). Hillsdale: Lawrence Erlbaum.
20. Conzelmann, A. (2001). Sport und Persönlichkeitsentwicklung. Möglichkeiten und Grenzen von Lebenslaufanalysen. Schorndorf: Hofmann.

21. Côté, J., Ericsson, K.A. \& Law, M.P. (2005). Tracing the development of athletes using retrospective interview methods: a proposed interview and validation procedure for reported information. Journal of Applied Sport Psychology, 17, 1-19.

22. Denk, H. \& Pache, D. (1996). Bewegung, Spiel und Sport im Alter. Band I: Bedürfnissituation Älterer Köln: Sport und Buch Strauß.

23. Eichberg, S. \& Rott, C. (2004). Sportverhalten im mittleren und höheren Erwachsenenalter. Bedingungsfaktoren für Kontinuität und Diskontinuität. Journal Public Health, 93-103.

24. Eichberg, S. (2003). Sportaktivität, Fitness und Gesundheit im Lebenslauf. Grundlagen für Prävention und Gesundheitsförderung aus der Sicht der Sportwissenschaft. Hamburg: Dr. Kovac.

25. Elder, G.H. jr. (1996). Human lives in changing societies: life course and development insights. In R.B. Cairns, G.H. Elder jr. \& E.J. Castello (Eds.), Developmental Sciences (pp. 31-62). Cambridge: University Press.

26. Frändin, K., Mellström, D., Sundh, V. \& Grimby, G. (1995). A life span perspective on patterns of physical activity and functional performance at the age of 76. Gerontology, 41, 109-120.

27. Frogner, E. (1991). Sport im Lebenslauf. Eine Verhaltensanalyse zum Breiten- und Freizeitsport. Stuttgart: Ferdinand Enke.

28. Hartmann-Tews, I., Tischer, U. \& Combrink, C. (2008a). Doing Gender und Doing Age im Kontext von Sport und Bewegung. Zeitschrift für Frauenforschung und Geschlechterstudien, 26 (2), 3251.

29. Hartmann-Tews, I., Tischer, U. \& Combrink, C. (2008b). Soziale Ungleichheit und Sport im Alter. In S. Nagel, T. Schlesinger, Y. Weigelt-Schlesinger \& R. Roschmann (Hrsg.), Sozialisation und Sport im Lebensverlauf. Jahrestagung der dvs-Sektion Sportsoziologie in Kooperation mit der dvs-Sektion Sportpädagogik vom 17. bis 19. September 2008 in Chemnitz (Schriften der Deutschen Vereinigung für Sportwissenschaft, 180, S. 107-108). Hamburg: Czwalina.

30. Hinsching, J. (1998). Der Bereich „Freizeit- und Erholungssport" im , ausdifferenzierten“ Sport der DDR. In J. Hinsching (Hrsg.), Alltagssport in der $D D R$ (S. 15-33). Aachen: Meyer und Meyer.

31. Hurrelmann, K. (1998). Gesundheitswissenschaftliche Ansätze in der Sozialisationsforschung. In K. Hurrelmann \& D. Ulrich (Hrsg.), Handbuch der Sozialisationsforschung (5. neu ausg. Aufl.) (S. 189213). Weinheim: Beltz.

32. Jekauc, D., Woll, A., Tittlbach, S., \& Bös, K. (2008). Stabilität der körperlich-sportlichen Aktivität im mittleren Erwachsenenalter. In M. Knoll \& A. Woll (Hrsg.), Sport und Gesundheit in der Lebensspanne. Jahrestagung der dvs-Kommission Gesundheit vom 10.-11. April 2008 in Bad Schönborn (Schriften der Deutschen Vereinigung für Sportwissenschaft, 174, S. 247-254). Hamburg: Czwalina.

33. Kemper, H.C.G., De Vente, W., Van Mechelen, W. \& Twisk, J.W.R. (2001). Adolescent motor skill and performance: is physical activity in adolescence related to adult physical fitness? American Journal of Human Biology, 13, 180-189.
34. Kirjonen, J., Telama, R., Luukkonen, R., Käärï, S., Kaila-Kangas, L. \& Leino-Arjas, P. (2006). Stability and prediction of physical activity in 5-, 10-, and 28-year-follow-up studies among industrial employees. Scandinavian Journal of Medicine \& Science in Sports, 16, 201-208.

35. Kjønniksen, L., Anderssen, N. \& Wold, B. (2009). Organized youth sport as a predictor of physical activity in adulthood. Scandinavian Journal of Medicine \& Science in Sports, 19, 646-654.

36. Klein, T. (2009). Determinanten der Sportaktivität und der Sportart im Lebenslauf. Kölner Zeitschrift für Soziologie und Sozialpsychologie, 61, 1-32.

37. Kristensen, P.L., Møller, N.C., Korsholm, L., Wedderkopp, N., Andersen, L.B. \& Froberg, K. (2008). Tracking of objectively measured physical activity from childhood to adolescence: The European youth heart study. Scandinavian Journal of Medicine \& Science in Sports, 18, 171-178.

38. Kuh, F.J.L. \& Cooper, C. (1992). Physical activity at 36 years: patterns and childhood predictors in longitudinal study. Journal of Epidemiology and Community Health, 46, 114-119.

39. Lamprecht, M. \& Stamm, H. (1995). Soziale Differenzierung und soziale Ungleichheit im Breitenund Freizeitsport. Sportwissenschaft, 25, 265284.

40. Lamprecht, M. \& Stamm, H. (2001). Sport in der zweiten Lebenshälfte. Analysen zum Seniorensport in der Schweiz. Magglingen: Bundesamt für Sport BASPO.

41. Lamprecht, M., Fischer, A. \& Stamm, H. (2008). Sport Schweiz 2008. Das Sportverhalten der Schweizer Bevölkerung. Magglingen: Bundesamt für Sport BASPO.

42. Lippke, S., Schwarzer, R. \& Fuchs, R. (2001). Erfolgreiches Altern durch Sport? Eine Metaanalyse. In R. Seiler (Hrsg.), Sportpsychologie: Anforderungen, Anwendungen, Aus- wirkungen. Internationale Fachtagung für Sportpsychologie 24. bis 26. Mai 2001 Magglingen, Schweiz (S. 155-157). Köln: Bps-Verlag.

43. Malina, R.M. (2001). Physical activity and fitness: pathways from childhood. American Journal of Human Biology, 13, 162-172.

44. Mayer, K.U. (1990). Lebensläufe und sozialer Wandel. Anmerkungen zu einem Forschungsprogramm. In K.U. Mayer (Hrsg.), Lebensverläufe und sozialer Wandel (Kölner Zeitschrift für Soziologie und Sozialpsychologie, Sonderheft 31/1990, S. 721). Opladen: Westdeutscher Verlag.

45. McMurray, R.G., Harrell, J.S., Bangdiwala, S.I. \& Hu, J. (2000). Tracking of Physical Activity and Aerobic Power from Childhood through Adolescence. Medicine \& Science in Sports \& Exercise, 35 (11), 1914-1922.

46. Möller, J. (1999). Sport im Alter. Sportwissenschaft, 29 (4), 440-454.

47. Nagel, M. (2003). Soziale Ungleichheiten im Sport. Aachen: Meyer und Meyer.

48. Nagel, S. \& Conzelmann, A. (2006). Zum Einfluss der Hochleistungssport-Karriere auf die Berufskarriere - Chancen und Risiken. Sport und Gesellschaft, 3, 237-261.

49. Nagel, S. (2002). Medaillen im Sport - Erfolg im Beruf? Berufskarrieren von Hochleistungssportlerinnen und Hochleistungssportlern. Schorndorf: Hofmann.

50. Pache, D. (2003). Die gegenwärtige Situation des Sports der Älteren. In H. Denk, D. Pache \& H.-J, Schaller (Hrsg.), Handbuch Alterssport (S. 67-74). Schorndorf: Hofmann. 
51. Pahmeier, I. (1994). Drop-out und Bindung im Breiten- und Gesundheitssport: günstige und ungünstige Bedingungen für eine Sportpartizipation. Sportwissenschaft, 24, 117-150.

52. Pfeiffer, K.A., Dowda, M., Dishman, R.K., Mclver, K.L., Sirard, J.R., Ward, D.S. \& Pate, R.R. (2006). Sport participation and physical activity in adolescent females across a four-year period. Journal of Adolescent Health, 39, 523-529.

53. Pitsch, W. (2005).,BBauchbare" und "unbrauchbare "Verkürzungen bei der Sportstättenentwicklungsplanung. Sportwissenschaft, 35 (3), 310 331.

54. Reimer, M. \& Matthes, B. (2007). Collecting event histories with True Tales: Techniques to improve autobiographical recall problems in standardized interviews. Quality and Quantity, 41 (5), 711-735.

55. Ropponen, A., Levälahti, E., Simonen, R., Videman, T. \& Battié, M.C. (2001). Repeatability of lifetime exercise reporting. Scandinavian Journal of Medicine and Science in Sports, 11, 185-192.

56. Scheerder, J., Vanreusel, B. \& Taks, M. (2005). Stratification patterns of active sport involvement among adults: Social change and persistence. International Review for the Sociology of Sport, 40 (2), 139-162.

57. Scheerder, J., Vanreusel, B., Taks, M. \& Renson, R. (2002). Social sports stratification in Flanders 1969-1999: Intergenerational reproduction of social inequalities? International Review for the Sociology of Sport, 37 (2), 219-245.

58. Schnell, R, Hill, P.B. \& Esser, E. (2008). Methoden der empirischen Sozialforschung. München: Oldenbourg.

59. Tammelin, T., Näyhä, S., Hills, A.P. \& Järvelin, M.-R. (2003). Adolescent participation in sports and adult physical activity. American Journal of Preventive Medicine, 24 (1), 22-28.

60. Taylor, W.C., Blair, S.N., Cummigs, S.S., Wun, C.C. \& Malina, R.M. (1999). Childhood and adolescent physical activity patterns and adult physical activity. Medicine \& Science in Sports \& Exercise, 31 (1), 118-123.

61. Telama, R., Leskinen, E. \& Yang, X. (1996). Stability of habitual physical activity and sport participation: a longitudinal tracking study. Scandinavian Journal of Medicine \& Science in Sports, 6, 371378.

62. Telama, R., Yang, X., Laakso, L. \& Viikari, J. (1997). Physical activity in childhood and adolescence as predictor of physical activity in young adulthood. American Journal of Preventive Medicine, 13 (4), 317-323.

63. Thiel, A., Huy, C. \& Gomolinsky, U. (2008). Alterssport in Baden-Württemberg - Präferenzen, Motive und Settings für die Sportaktivität in der Generation 50+. Deutsche Zeitschrift für Sportmedizin, 59 (7-8), 163-167.

64. Trillitzsch, M. (2004). Die Entwicklung des kommerziellen Sportanbietermarktes in Ostdeutschland. Eine Untersuchung aus modernisierungsund transformationstheoretischer Perspektive. Hamburg: Kovac.

65. Trudeau, F., Laurencelle, L. \& Shephard, R.J. (2004). Tracking of physical activity from childhood to adulthood. Medicine \& Science in Sports \& Exercise, 36 (11), 1937-1943.

66. Twisk, J.W.R., Kemper, H.C.G. \& van Mechelen, W. (2000). Tracking of activity and fit ness and the relationship with cardiovascular disease risk factors. Medicine \& Science in Sports \& Exercise, 32 (8), 1455-1461.
67. Wagner, P. (2000). Aussteigen oder Dabeibleiben? Determinanten der Aufrechterhaltung sportlicher Aktivität in gesundheitsorientierten Sportprogrammen. Darmstadt: Wissenschaftliche Buchgesellschaft.

68. Woll, A. (2006). Sportliche Aktivität, Fitness und Gesundheit im Lebenslauf. Eine internationale Längsschnittstudie. Schorndorf: Hofmann.

\section{Haas mit Forschungspreis ausgezeichnet}

Stiftung würdigt Arbeiten des Sportwissenschaftlers

Mit dem Forschungspreis der Hilde-UlrichsStiftung für Parkinsonforschung wurde Professor Dr. Christian T. Haas, Sportwissenschaftler und Forschungskoordinator im Fachbereich Gesundheit an der Hochschule Fresenius in Idstein, ausgezeichnet. Der mit 10.000 Euro bisher höchstdotierte Preis der Stiftung wurde von deren Vorsitzenden Hermann Terweiden im Rahmen einer Feierstunde in Schmitten verliehen.

Mit diesem Preis würdigt die Hilde-UlrichsStiftung für Parkinsonforschung Haas' Forschungsarbeiten für das Wohl von Parkinsonkranken und Menschen mit anderen neurologischen Krankheiten - wie beispielsweise Multipler Sklerose. Mit vielen eigenen und Untersuchungsergebnissen anderer hochkarätiger Wissenschaftler habe Haas eindrucksvoll belegt, dass Parkinsonkranke die Chance haben, mit spezifischem Kraft-, Ausdauerund Koordinationstraining die motorischen Einschränkungen erheblich zu reduzieren, begründete die Stiftung ihre Entscheidung. Das Preisgeld wird Haas in seine weitere Forschung investieren, so dass beispielsweise praktikable Trainingsanleitungen für Parkinsonkranke und andere neurologische Erkrankungen umgesetzt werden können. Die Hilde-Ulrichs-Stiftung für Parkinsonforschung wurde am 1. Welt-Parkinson-Tag am 11. April 1997 in Florstadt gegründet und wird ehrenamtlich überwiegend von Parkinsonkranken geführt. Die gemeinnützige Organisation fördert schwerpunktmäßig die Grundlagenforschung und nichtmedikamentöse Behandlungsverfahren. Auch Beratung und Information über die neurologische Krankheit Morbus Parkinson sind wichtige Ziele. 